\title{
Exploring the Higgs sector of the MRSSM with a light scalar
}

\author{
Philip Diessner, ${ }^{a, 1}$ Jan Kalinowski, ${ }^{b}$ Wojciech Kotlarski ${ }^{a, b}$ and Dominik Stöckinger ${ }^{a}$ \\ ${ }^{a}$ Institut für Kern- und Teilchenphysik, TU Dresden, \\ 01069 Dresden, Germany \\ ${ }^{b}$ Faculty of Physics, University of Warsaw, \\ Pasteura 5, 02093 Warsaw, Poland \\ E-mail: philip.diessner@mailbox.tu-dresden.de, \\ jan.kalinowski@fuw.edu.pl, wojciech.kotlarski@fuw.edu.pl, \\ dominik.stoeckinger@tu-dresden.de
}

ABSTRACT: In a recent paper we showed that the Minimal R-symmetric Supersymmetric Standard Model (MRSSM) can accommodate the observed $125 \mathrm{GeV}$ Higgs boson as the lightest scalar of the model in agreement with electroweak precision observables, in particular with the $\mathrm{W}$ boson mass and $T$ parameter. Here we explore a scenario with the light singlet (and bino-singlino) state in which the second-lightest scalar takes the role of the SM-like boson with mass close to $125 \mathrm{GeV}$. In such a case the second-lightest Higgs state gets pushed up via mixing already at tree-level and thereby reducing the required loop correction. Unlike in the NMSSM, the light singlet is necessarily connected with a light neutralino which naturally appears as a promising dark matter candidate. We show that dark matter and LHC searches place further bounds on this scenario and point out parameter regions, which are viable and of interest for LHC Run II and upcoming dark matter experiments.

KEYWORDS: Supersymmetry Phenomenology

ArXiv ePrint: 1511.09334

${ }^{1}$ Corresponding author. 


\section{Contents}

1 Introduction 1

2 Higgs constraints 3

2.1 Scenario description 3

$\begin{array}{ll}2.2 & \text { Quantitative analysis and comparison to experiment } 6\end{array}$

3 LHC constraints $\quad 9$

$\begin{array}{lll}3.1 & \text { Constraints from slepton searches } & 10\end{array}$

3.2 Qualitative description of the MRSSM chargino/neutralino sector 10

$\begin{array}{lll}3.3 & \text { Setup for recasting LHC limits for the MRSSM } & 11\end{array}$

$\begin{array}{lll}3.4 & \text { Light staus and light charginos } & 13\end{array}$

$\begin{array}{ll}\text { 3.5 General investigation of light charginos } & 14\end{array}$

4 Dark matter constraints $\quad 16$

$\begin{array}{ll}4.1 \text { Relic density } & 17\end{array}$

$\begin{array}{lll}4.2 & \text { Direct detection } & 18\end{array}$

$\begin{array}{llr}5 & \text { Summary and conclusions } & 20\end{array}$

A MRSSM mass matrices at tree level $\quad 24$

\section{Introduction}

After the discovery of a Standard Model (SM)-like Higgs boson at the LHC [1-3], it remains an open question whether there are additional scalar particles, possibly even with smaller mass. Supersymmetric models always predict the existence of additional scalars, and the possibility of light scalars has been explored both in the minimal supersymmetric standard model (MSSM) [4-6] and in its extensions, such as the NMSSM which contains a gauge singlet field $[7,8]$. In the NMSSM a light singlet-like scalar is motivated since it pushes the tree-level value of the SM-like Higgs boson mass up towards the observed value, reducing the need for large loop corrections [9-21]. The interplay of the Higgs sector and dark matter results in the MSSM and NMSSM was further discussed in [22-28].

In the present paper we consider the possibility of a light, singlet-like scalar in the minimal R-symmetric supersymmetric model, the MRSSM [29]. We show that it is necessarily connected with a very light neutralino LSP, and we explore the phenomenology of the Higgs sector and the related chargino/neutralino sector, taking into account collider and dark matter constraints.

The MRSSM is quite distinct from the usual MSSM and NMSSM and is motivated in several ways. Its essential feature is an exact, global U(1) R-symmetry [30, 31], under which 
SM-fields and superpartners have different charges. This symmetry is stronger than Rparity, and it forbids Majorana masses for gauginos, the MSSM Higgsino mass parameter $\mu$, and all left-right sfermion mixings. As a result, several of the most important experimental constraints on supersymmetry are alleviated: contributions to CP- and flavour-violating observables are suppressed even in presence of flavour violation in the sfermion sector [29, 32 , and the production cross section for squarks is reduced, making squarks below the $\mathrm{TeV}$ scale generically compatible with LHC data [33].

The most exciting implication of R-symmetry from the point of view of scalars is that additional scalar fields are not ad hoc but are enforced by an $\mathrm{N}=2$ supersymmetric structure of the gauge/gaugino sector: for each gauge group factor, there are gauge fields, Dirac instead of Majorana gauginos, and scalar fields in the adjoint representation. The MRSSM therefore contains sgluons - colour-octet scalars, a scalar SU(2) triplet, and a scalar singlet. This scalar singlet behaves quite differently and is more strongly connected to the other sectors of the theory than the singlet of the NMSSM.

Previous phenomenological investigations of the MRSSM have not focused on a light singlet, but it has been shown that models with R-symmetry and/or Dirac gauginos contain promising dark matter candidates [34-36], and the collider physics of the extra, non-MSSMlike states has been studied [37-45].

Recently in refs. [46, 47] the lightest (SM-like) Higgs boson mass has been computed at the one-loop and leading two-loop level and the W-boson mass has been computed taking into account tree-level corrections to $\rho$ from the triplet vacuum expectation value and full one-loop corrections to muon decay. It was shown that the measured value of the Higgs boson mass at the LHC can be accommodated in the MRSSM even for top squarks as light as $1 \mathrm{TeV}$. The outcome of these calculations was not obvious since in the MRSSM the lightest Higgs boson tree-level mass is typically reduced compared to the MSSM due to mixing with the additional scalars, and the loop corrections cannot be enhanced by stop mixing. However, the new fields and couplings can give rise to the necessary large loop contributions to the Higgs mass without generating too large a contribution to the W-boson mass. For a similar analysis see ref. [48], where also a welcome reduction of the level of fine-tuning was found.

The plan of the present paper is as follows. In section 2 we remind the reader of the relevant model details and will describe how demanding a light singlet scalar constrains the parameter space of the model. Sections 3 and 4 study how LHC and dark matter bounds, respectively, are affected by these constraints. To highlight features of different regions of parameters, which are also in agreement with LHC and dark matter searches, we define the new Benchmark points given in table 1. They feature many states well below $1 \mathrm{TeV}$, including the top squark masses. The combination of all different experimental constraints on the parameter space of the MRSSM is summarized in section 5 which also contains our conclusions. 


\begin{tabular}{|lrrr|}
\hline & BMP4 & BMP5 & BMP6 \\
\hline $\tan \beta$ & 40 & 20 & 6 \\
$B_{\mu}$ & $200^{2}$ & $200^{2}$ & $500^{2}$ \\
$\lambda_{d}, \lambda_{u}$ & $0.01,-0.01$ & $0.0,-0.01$ & $0.0,0.0$ \\
$\Lambda_{d}, \Lambda_{u}$ & $-1,-1.2$ & $-1,-1.15$ & $-1,-1.2$ \\
\hline$M_{B}^{D}$ & 50 & 44 & 30 \\
$m_{S}^{2}$ & $30^{2}$ & $40^{2}$ & $80^{2}$ \\
$m_{R_{u}}^{2}, m_{R_{d}}^{2}$ & & $1000^{2}, 700^{2}$ & \\
$\mu_{d}, \mu_{u}$ & 130,650 & 400,550 & 550,550 \\
$M_{W}^{D}$ & 600 & 500 & 400 \\
$M_{O}^{D}$ & & 1500 & \\
$m_{T}^{2}, m_{O}^{2}$ & & $3000^{2}, 1000^{2}$ & \\
\hline$m_{Q ; 1,2}^{2}, m_{Q ; 3}^{2}$ & $1500^{2}, 700^{2}$ & $1300^{2}, 700^{2}$ & $1400^{2}, 700^{2}$ \\
$m_{D ; 1,2}^{2}, m_{D ; 3}^{2}$ & $1500^{2}, 1000^{2}$ & $1300^{2}, 1000^{2}$ & $1400^{2}, 1000^{2}$ \\
$m_{U ; 1,2}^{2}, m_{U ; 3}^{2}$ & $1500^{2}, 700^{2}$ & $1300^{2}, 700^{2}$ & $1400^{2}, 700^{2}$ \\
$m_{L ; 1,2}^{2}, m_{E ; 1,2}^{2}$ & $800^{2}, 800^{2}$ & $1000^{2}, 1000^{2}$ & $500^{2}, 350^{2}$ \\
$m_{L ; 3,3}^{2}, m_{E ; 3,3}^{2}$ & $800^{2}, 136^{2}$ & $1000^{2}, 1000^{2}$ & $500^{2}, 95^{2}$ \\
\hline$m_{H_{d}}$ & $1217^{2}$ & $211^{2}$ & $1042^{2}$ \\
$m_{H_{u}}$ & $-\left(767^{2}\right)$ & $-\left(207^{2}\right)$ & $-(201)^{2}$ \\
$v_{S}$ & -64.9 & -42.5 & -56.1 \\
$v_{T}$ & -1.08 & -1.2 & -1.1 \\
\hline
\end{tabular}

Table 1. Benchmark points for the scenario discussed here. Dimensionful parameters are given in $\mathrm{GeV}$ or $\mathrm{GeV}^{2}$, as appropriate.

\section{Higgs constraints}

\subsection{Scenario description}

The essential field content and parameters of the MRSSM can be read off from the superpotential

$$
\begin{aligned}
W= & \mu_{d} \hat{R}_{d} \cdot \hat{H}_{d}+\mu_{u} \hat{R}_{u} \cdot \hat{H}_{u}+\Lambda_{d} \hat{R}_{d} \cdot \hat{T} \hat{H}_{d}+\Lambda_{u} \hat{R}_{u} \cdot \hat{T} \hat{H}_{u} \\
& +\lambda_{d} \hat{S} \hat{R}_{d} \cdot \hat{H}_{d}+\lambda_{u} \hat{S} \hat{R}_{u} \cdot \hat{H}_{u}-Y_{d} \hat{d} \hat{q} \cdot \hat{H}_{d}-Y_{e} \hat{e} \hat{l} \cdot \hat{H}_{d}+Y_{u} \hat{u} \hat{q} \cdot \hat{H}_{u} .
\end{aligned}
$$

The MSSM-like fields are the Higgs doublet superfields $\hat{H}_{d, u}$ and the quark and lepton superfields $\hat{q}, \hat{u}, \hat{d}, \hat{l}, \hat{e}$. The Yukawa couplings are the same as in the MSSM. The new fields are the doublets $\hat{R}_{d, u}$, which contain the Dirac mass partners of the higgsinos and the corresponding Dirac higgsino mass parameters are denoted as $\mu_{d, u}$. The singlet, the $\mathrm{SU}(2)$-triplet and the color-octet chiral superfields, $\hat{S}, \hat{T}$ and $\hat{O}$, contain the Dirac mass partners of the usual gauginos. The superpotential contains Yukawa-like trilinear terms involving the new fields; the associated parameters are denoted as $\lambda_{d, u}$ for the terms 
involving the singlet and $\Lambda_{d, u}$ for the terms involving the triplet; terms involving the octet are not allowed by R-symmetry. Further important parameters of the MRSSM are the Dirac mass parameters $M_{B, W, O}^{D}$ for the $\mathrm{U}(1), \mathrm{SU}(2)_{L}$ and $\mathrm{SU}(3)_{c}$ gauginos, respectively, the soft scalar mass parameters $m_{S, T, O}^{2}$ for the singlet, triplet and octet states, the soft mass parameters $m_{H_{d}, H_{u}, R_{d}, R_{u}}^{2}$ for the Higgs and R-Higgs bosons, and the standard $B_{\mu}$ parameter and sfermion mass parameters, while the trilinear sfermion couplings are forbidden by R-symmetry. The explicit form of the soft SUSY breaking potential is given in ref. [46].

We begin the discussion with the neutral Higgs sector of the MRSSM. There are four complex (electrically and R-charge) neutral scalar fields, which can mix. Their real and imaginary parts, denoted as $\left(\phi_{d}, \phi_{u}, \phi_{S}, \phi_{T}\right)$ and $\left(\sigma_{d}, \sigma_{u}, \sigma_{S}, \sigma_{T}\right)$, correspond to the two MSSM-like Higgs doublets $H_{d, u}$, and the $N=2$ scalar superpartners of the hypercharge and $\mathrm{SU}(2)_{L}$ gauge fields, $S$ and $T$. Since their vacuum expectation values, denoted as $v_{d, u, S, T}$ respectively, are assumed real, the real and imaginary components do not mix, and the mass-square matrix breaks into two $4 \mathrm{x} 4$ sub-matrices.

In the pseudo-scalar sector the MSSM-like states $\left(\sigma_{d}, \sigma_{u}\right)$ do not mix with the singlettriplet states $\left(\sigma_{S}, \sigma_{T}\right)$ and the mass-square matrix breaks further into two $2 \times 2$ sub-matrices, see appendix A. Therefore the neutral Goldstone boson and one of the pseudo-scalar Higgs bosons $A$ with $m_{A}^{2}=2 B_{\mu} / \sin 2 \beta$ appear as in the MSSM. On the other hand, in the $\left(\sigma_{S}, \sigma_{T}\right)$ sector the mixing is negligible for a heavy triplet mass $m_{T}$ and the lightest pseudoscalar state is almost a pure singlet with its mass given by the soft parameter $m_{S}$. Therefore, to a good approximation it decouples and is of no interest for the following discussion.

In the scalar sector all fields mix and the full $4 \times 4$ tree-level mass-square matrix $\mathcal{M}^{\phi}$ in the weak basis $\left(\phi_{d}, \phi_{u}, \phi_{S}, \phi_{T}\right)$ has been given explicitly in ref. [46]. It is diagonalized by a unitary rotation $Z^{H} \mathcal{M}^{\phi} Z^{H^{\dagger}}$ to the mass-eigenstate basis $\left(H_{1}, H_{2}, H_{3}, H_{4}\right)$, ordered with increasing mass. For the qualitative discussion we consider the limit in which the triplet decouples due to a high soft breaking mass term $m_{T}$ (a necessary condition to suppress the tree-level triplet contribution to the $\mathrm{W}$ boson mass and $\rho$ parameter), and in which the pseudoscalar Higgs mass $M_{A}$ and the value of $\tan \beta=v_{u} / v_{d}$ become large. In this limit the SM-like Higgs boson is dominantly given by the up-type field $\phi_{u}$, and to highlight the main effect of the mixing in the light-scalar scenario it is enough to recall the most relevant part of the mass-square matrix, namely the $2 \times 2$ sub-matrix corresponding to the $\left(\phi_{u}, \phi_{S}\right)$ fields only, which reads

$$
\mathcal{M}_{u, S}^{\phi}=\left(\begin{array}{cc}
m_{Z}^{2}+\Delta m_{\mathrm{rad}}^{2} & v_{u}\left(\sqrt{2} \lambda_{u} \mu_{u}^{\mathrm{eff},-}+g_{1} M_{B}^{D}\right) \\
v_{u}\left(\sqrt{2} \lambda_{u} \mu_{u}^{\mathrm{eff},-}+g_{1} M_{B}^{D}\right) & 4\left(M_{B}^{D}\right)^{2}+m_{S}^{2}+\frac{\lambda_{u}^{2} v_{u}^{2}}{2}
\end{array}\right),
$$

where we have included the dominant radiative correction $\Delta m_{r a d}^{2}$ to the diagonal element of the doublet field $\phi_{u}$, and we have used the abbreviations

$$
\mu_{i}^{\mathrm{eff}, \pm}=\mu_{i}+\frac{\lambda_{i} v_{S}}{\sqrt{2}} \pm \frac{\Lambda_{i} v_{T}}{2}, \quad i=u, d .
$$

The parameters $M_{B}^{D}, \mu_{u}$ and $\lambda_{u}$ appear in the scalar potential due to supersymmetry. ${ }^{1}$ From this approximation to the mass-square matrix we can draw several immediate

\footnotetext{
${ }^{1}$ In ref. [49] it was recently shown that Dirac masses could also be generated without contributions to the scalar sector. Here, we keep with the usual D-term generation of these mass terms for the MRSSM.
} 
conclusions regarding the four most important parameters in the light-singlet scenario in which $m_{H_{1}}<m_{H_{2}} \approx 125 \mathrm{GeV}$.

a) Singlet soft mass $m_{S}^{2}$.

The soft breaking parameter $m_{S}^{2}$ appears in the diagonal element for the singlet field $\phi_{S}$ in the mass matrix (2.2). To ensure that the lightest scalar state is a singlet-like a direct upper limit can be put on this parameter:

$$
m_{S}^{2}<m_{Z}^{2}+\Delta m_{\mathrm{rad}}^{2}
$$

Therefore the numerical value of $m_{S}^{2}$ has to be limited to be below $\approx(120 \mathrm{GeV})^{2}$

b) Dirac bino-singlino mass $M_{B}^{D}$.

Due to supersymmetry, the Dirac mass parameter $M_{B}^{D}$ appears also in the singlet diagonal element, and moreover in the off-diagonal elements as well. Hence, a direct upper limit can be derived

$$
\left(M_{B}^{D}\right)^{2}<\frac{m_{Z}^{2}+\Delta m_{r a d}^{2}}{4} \approx(60 \mathrm{GeV})^{2},
$$

similar to the one on $m_{S}^{2}$ (however stronger) to keep the singlet-diagonal element smaller than the doublet one. Of course, the bino-singlino mass parameter appears also in the neutralino mass matrix and is the dominant parameter that determines the LSP mass in the light singlet/bino-singlino scenario. Therefore constraints from dark matter and direct LHC searches for neutralinos will have a strong impact on the Higgs sector.

c) Higgsino mass $\mu_{u}$.

The higgsino mass parameter $\mu_{u}$ appears in the off-diagonal element of the mass matrix in eq. (2.2) in combination with $\lambda_{u}$. However $\mu_{u}$ also enters as a parameter in the chargino/neutralino sector, and it is strongly limited from below by the direct chargino searches and dark matter constraints. We recall that $\mu_{u}$ has a strong influence on the loop corrections $\Delta m_{\text {rad }}^{2}$ to the doublet component $\phi_{u}$, so it can affect the mixing additionally through that diagonal element.

d) Yukawa-like parameter $\lambda_{u}$.

The Yukawa-like parameter $\lambda_{u}$ multiplies the higgsino mass parameter $\mu_{u}$ in the offdiagonal element of eq. (2.2). The off-diagonal element cannot be too large in order to avoid a negative determinant of the mass matrix and thus tachyonic states. In the simple limit $v_{S}, m_{Z} \ll \mu_{u}$, avoiding tachyons leads to the following bound on $\lambda_{u}$ :

$$
\left|\lambda_{u}+\frac{g_{1} M_{B}^{D} v_{u}}{\sqrt{2} \mu_{u} v_{u}}\right|<\frac{m_{Z} \sqrt{4\left(M_{B}^{D}\right)^{2}+m_{S}^{2}}}{\sqrt{2} \mu_{u} v_{u}}
$$

There can be a cancellation between $\lambda_{u}$ and the $M_{B}^{D}$-term, but since $\mu_{u}$ is much larger than all other appearing mass parameters, the value of $\lambda_{u}$ is necessarily very small. 
In summary, these simple considerations lead to the following promising parameter hierarchies for a light singlet scenario:

$$
m_{S}, M_{B}^{D}<m_{Z}<\mu_{u}, \quad\left|\lambda_{u}\right| \ll 1 .
$$

\subsection{Quantitative analysis and comparison to experiment}

In our phenomenological investigations we will of course not use the approximations made before but we will always use the most precise available expressions including higher order corrections. This is done using the Mathematica [50] package SARAH [51-56] and SPheno [57, 58]. This approach was cross-checked, as described in refs. [46, 47], using FlexibleSUSY[59], FeynArts and FormCalc [62-64].

Figure 1 shows a quantitative analysis of the mixing of the two lightest Higgs states, as a function of the two relevant parameters $m_{S}$ (left column) and $M_{B}^{D}$ (right column). The other parameters are chosen as in BMP5, given in table 1 . They satisfy the hierarchy of eq. (2.7); the precise parameter choices will be motivated in the subsequent sections.

The first two lines in the figure show the masses of the two lightest Higgs states (computed to two-loops as described in ref. [47]) and the singlet component of these two states, measured by the square of the corresponding mixing matrix elements $\left(Z_{H_{1}, \phi_{S}}^{H}\right)^{2}$ and $\left(Z_{H_{2}, \phi_{S}}^{H}\right)^{2}$. As expected from the discussion above, as long as the approximate inequalities eq. (2.4) and eq. (2.5) are satisfied, the lightest state is significantly lighter than $m_{Z}$ and has a high singlet component. Once $m_{S}$ or $M_{B}^{D}$ become heavier, the lightest state becomes mainly a doublet-like (and hence SM-like) and its mass approaches a limit, which is here below $120 \mathrm{GeV}$. Which of the parameter regions is phenomenologically viable will be determined below.

The third line of plots in figure 1 quantifies one of the major motivations for the light singlet scenario - the enhancement of the SM-like Higgs boson mass due to the tree-level push resulting from mixing with the scalar state. The plots show the difference of the actual value of this mass and the value in a reference case, in which all Higgs bosons except the SM-like one are very heavy. In the light-singlet case, the upward shift can amount to more than $10 \mathrm{GeV}$. The upward shift is particularly strong for non-vanishing Dirac mass $M_{B}^{D}$, since this parameter also appears in the off-diagonal element of the mass matrix eq. (2.2).

Now we compare the light-singlet scenario illustrated in figure 1 with experimental data. It is clear that there are two kinds of direct constraints on the two lightest scalars and their mixing. On the one hand, there must be a SM-like state which can be identified with the Higgs boson observed at LHC, with mass compatible with the observed value and with sufficiently small singlet mix-in in order to agree with the observed Higgs signal strengths and branching ratios. On the other hand, the state that is lighter than the one observed at the LHC must be sufficiently singlet-like in order to evade limits from direct searches for light scalars, especially the LEP searches. Roughly speaking, for light singlet masses these constraints restrict the mixing to around $\left(Z_{H_{1}, \phi_{u}}^{H}\right)^{2}<0.02$, except for the region, where LEP saw an upward fluctuation. Here the mixing is restrained to $\left(Z_{H_{1}, \phi_{u}}^{H}\right)^{2}<0.15$.

Both types of constraints can be precisely analyzed with the help of HiggsBounds-4.2 and HiggsSignals-1.4, respectively [65-70]. Here, we pass the effective couplings written 

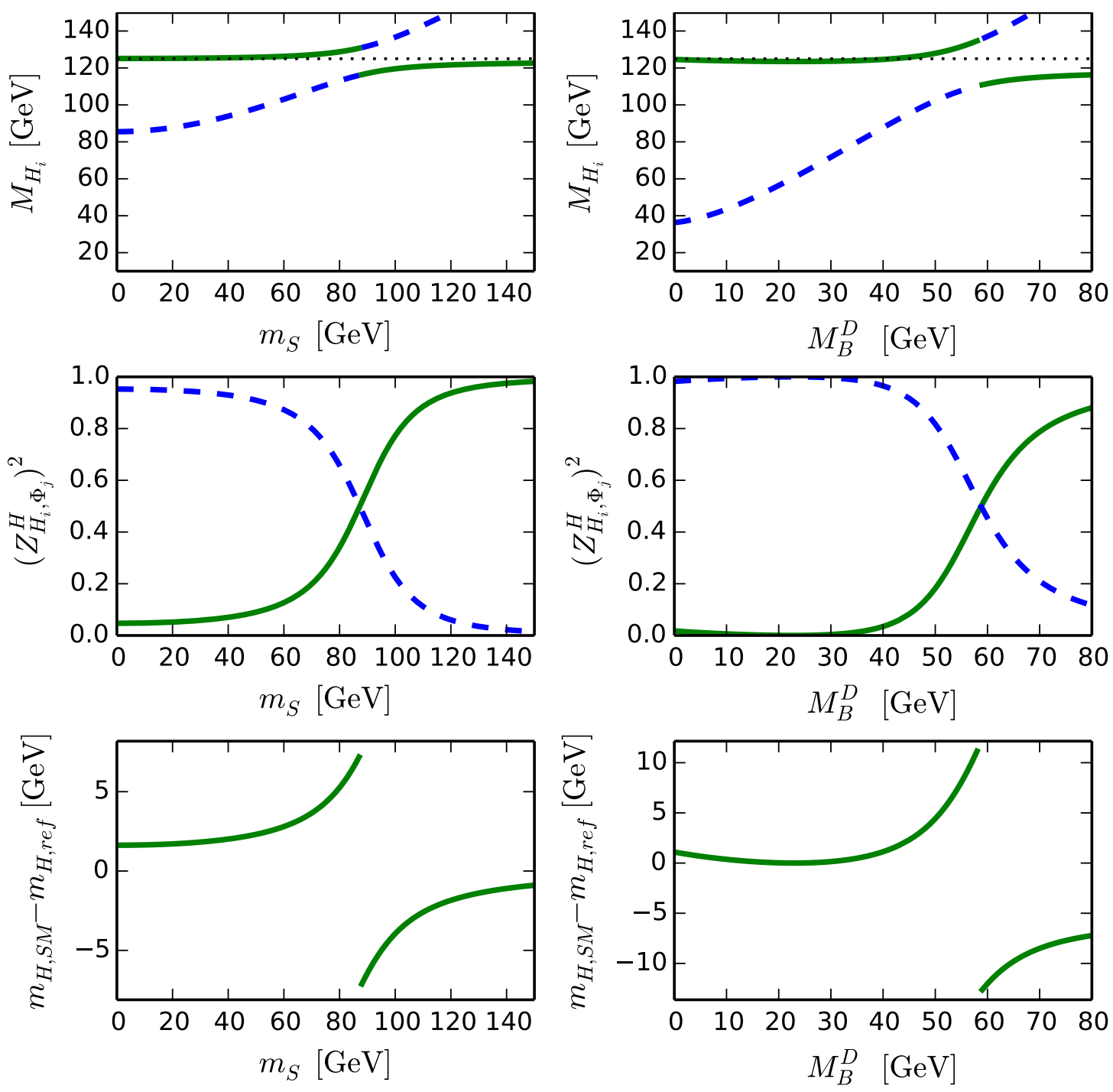

Figure 1. Level crossing of lightest and second-lightest Higgs state for BMP5, depending on the dimensionful parameter $m_{S}\left(M_{B}^{D}\right)$ on the left (right). Top row shows the masses of the lightest and second-lightest mass eigenstate, the green full line is more SM-like, while the blue dashed line is singlet-like. The second line shows the mixing parameter as described in the text. The blue dashed line is the singlet content of the lightest mass eigenstate, the green full line the doublet content. The bottom row gives the relative mass shift compared to a reference mass, where the mixing between singlet and doublet vanishes.

by SPheno to both tools for each parameter point studied. With HiggsBounds the option $L a n d H$ is used to check the MRSSM Higgs sector against all experimental constraints included with the program. For HiggsSignals all peak observables available are used (option latestresults and peak), the mass uncertainties are described using a Gaussian shape and we estimate the uncertainty of the SM-like Higgs boson mass with $3 \mathrm{GeV}$.

The interpretation of the HiggsBounds and HiggsSignals results is done in a simplified way. A parameter point is excluded by HiggsBounds by using the 95\% CL limit of its 

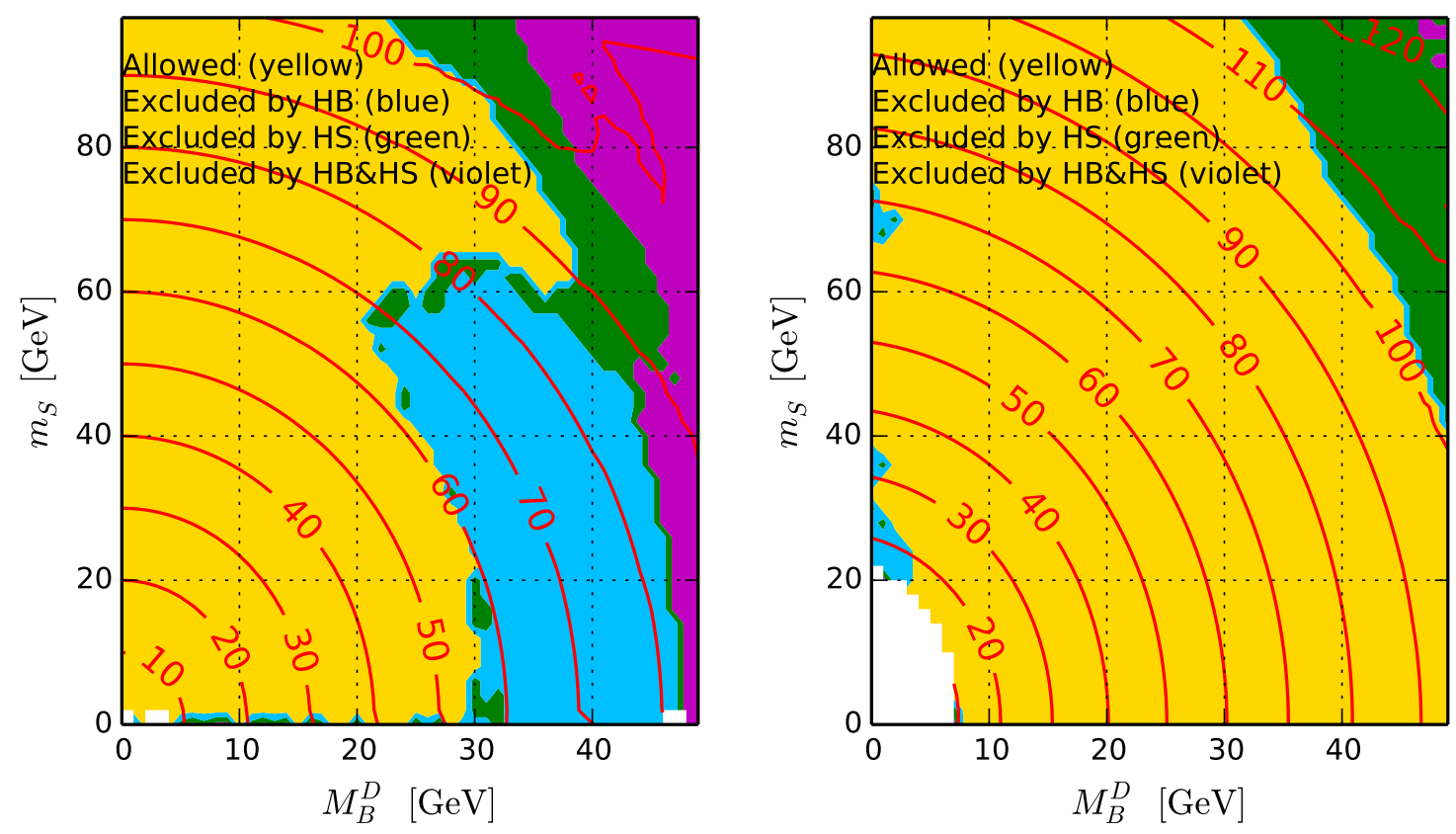

Figure 2. Exclusion plot using HiggsSignals-1.3 and HiggsBounds-4.2; Higgs masses are calculated at two loops. Scanned over $\Lambda_{u}$ and projected onto the plane. If there is one $\Lambda_{u}$ for a point giving non-exclusion, it is shown as non-excluded. Scanning from $-1.5<\Lambda_{u}<0$, while $\lambda_{u}=0 /-0.01$ (left,right). Red contours show the lightest Higgs boson mass.

output and by HiggsSignals when its approximately calculated p-value is smaller than 0.05. A point is excluded by both when both constraints apply. For a more complete statistical treatment of extended Higgs sectors containing light singlet scenarios for the singlet-extended SM and NMSSM see refs. [71] and [72], respectively. In the case of the MRSSM this approach is left for future work.

Figure 2 shows the resulting excluded and allowed regions in the plane of $m_{S}$ and $M_{B}^{D}$ for two different values of $\lambda_{u}$. The parameter $\Lambda_{u}$ is adjusted to ensure the correct mass of the observed Higgs boson; the remaining parameters are fixed as in BMP5. The combination of the two plots shows that complete range of $m_{S}$ and $M_{B}^{D}$ discussed before with simple approximations of the tree-level scalar mass matrix is viable, except of the region of level crossing and very strong mixing. We find that the light-singlet scenario can be realized in the MRSSM for $m_{S}<100 \mathrm{GeV}$ and $M_{B}^{D}<55 \mathrm{GeV}$. However, the two plots also show very high sensitivity to the small $\lambda_{u}$, which appears essentially in combination with the very small term $\propto M_{B}^{D} / \mu_{u}$, see (2.6). Changing it from zero to $(-0.01)$ leads to strong shifts of the allowed regions.

The main novel feature of the light-singlet scenario in the MRSSM is the restriction on the Dirac bino-singlino mass $M_{B}^{D}$. This restriction has no counterpart e.g. in the NMSSM. Obviously this limit affects the neutralino sector. Combined with LEP bounds on chargino and neutralino production it leads to the conclusion that the LSP is a Dirac bino-singlino neutralino with mass related to $M_{B}^{D}$ and thus limited from above. This limit on the LSP mass will provide strong constraints from and correlations to LHC processes and dark matter studies. 


\begin{tabular}{|lrrr|}
\hline & BMP4 & BMP5 & BMP6 \\
\hline$m_{H_{1}}$ & 100 & 94 & 95 \\
$m_{H_{2}}$ & 125.8 & 125.5 & 125.8 \\
HiggsSignals p-value & 0.75 & 0.76 & 0.72 \\
Allowed by HiggsBounds & $\checkmark$ & $\checkmark$ & $\checkmark$ \\
$m_{W}$ & 80.384 & 80.392 & 80.404 \\
\hline
\end{tabular}

Table 2. Higgs sector observables: masses of the Higgs bosons, HiggsSignals p-value, HiggsBounds check and, for completeness, the $\mathrm{W}$ boson mass for the benchmark points. Dimensionful parameters are given in $\mathrm{GeV}$.

With these restrictions on $m_{S}$ and $M_{B}^{D}$, the decay of the $\mathrm{Z}$ and SM-like Higgs boson into the LSP and singlet-like Higgs boson, if kinematically allowed, needs to be sufficiently suppressed so that theoretical prediction of invisible width of both particles is in agreement with experiment. In the mass region of interest, $m_{H_{1}}<62 \mathrm{GeV}$, HiggsBounds puts very stringent limits on the singlet-doublet mixing. As the decay of the SM-like Higgs boson into the light (pseudo-)scalar is related to this mixing, contributions of these decay channels are strongly reduced for realistic scenarios. Decays of Z (Higgs) bosons to LSPs are induced by the couplings to the (R-)higgsino (higgsino+bino or R-higgsino+singlino) parts of the LSP. This is mainly mediated by gauge couplings and will be suppressed by the weak (R)higgsino-singlino-bino-mixing. We will show that it is also important for direct detection limits from dark matter searches. These searches put such a strong bound on the mixing so that the decay to the LSP will be automatically suppressed.

Table 2 summarizes the Higgs sector observables for our benchmark points, as well as the predicted $\mathrm{W}$ boson mass.

\section{LHC constraints}

As was discussed in the previous section, demanding the singlet state to give the lightest Higgs boson yields a direct upper limit on the Dirac bino mass $M_{B}^{D}$. A light bino-like neutralino, and more generally light weakly interacting particles, are promising in two respects. They might lead to observable signals at the LHC, and they might explain the observed dark matter relic density. At the same time, the light-singlet scenario is constrained by existing data from LHC and dark matter searches. In the present section we study the constraints from LHC in detail. We only consider constraints on weakly interacting particles, i.e. on charginos, neutralinos, and sleptons. All strongly interacting particles are not relevant for the electroweak and dark matter phenomenology studied here and are assumed to be heavy enough to evade limits.

So far the negative results of LHC searches for new particles have been interpreted within the MSSM, or a number of simplified models which include a pair of charginos, the neutralinos and gluinos are of the Majorana type, and left- and right-chiral sfermions that can mix. In general, care has to be taken in reinterpreting the LHC bounds in the context of the MRSSM, since the MRSSM differs from the MSSM in the number of degrees of 
freedom, the Dirac versus Majorana nature of neutralinos, the mixing patterns, and since some processes are forbidden in the MRSSM due to the conserved R-charge.

\subsection{Constraints from slepton searches}

We first discuss briefly the simplest case of constraints following from slepton searches. In both the MSSM and the MRSSM, sleptons can be produced in pairs through DrellYan processes. In the MRSSM, slepton left-right mixing vanishes, which is not an essential difference to the MSSM, where the mixing is typically assumed to be small. In both models, light sleptons will decay directly to the corresponding leptons and the bino-like neutralino LSP. In such a case the Dirac or Majorana nature of the LSP does not matter and, hence, the MSSM exclusion bounds for light sleptons can be applied directly to the MRSSM case. Recently the ATLAS Collaboration derived the exclusion limits in the $\left(m_{\tilde{\ell}_{L, R}}, m_{\tilde{\chi}_{1}^{0}}\right)$ parameter space from the analyses of the selectron and smuon pair-production processes, see figures 8 (a) and (b) of ref. [73]. ${ }^{2}$ These exclusion limits can be specialized to our case, in which eq. (2.5) implies an upper limit of $\approx 55 \mathrm{GeV}$ on the LSP mass. We then find that left-handed slepton masses below $\approx 300 \mathrm{GeV}$ are excluded. For the right-handed slepton masses the bound is somewhat weaker (around $250 \mathrm{GeV}$ ) due to smaller production cross section. Further, a small corner at a lower right-handed slepton mass $\sim 100 \mathrm{GeV}$ with the LSP masses in the range $40-55 \mathrm{GeV}$ is still allowed. With the current experimental reach the direct production of staus with the cross sections predicted by supersymmetry can not be excluded, see ref. [75].

The remainder of the present section is devoted to the more interesting case of neutralino and chargino searches.

\subsection{Qualitative description of the MRSSM chargino/neutralino sector}

The chargino and neutralino sectors of the MSSM and the MRSSM are quite different. In the MRSSM, the neutralinos are Dirac fermions composed of eight Weyl spinors $\xi_{i}=$ $\left(\tilde{B}, \tilde{W}^{0}, \tilde{R}_{d}^{0}, \tilde{R}_{u}^{0}\right)$ and $\zeta_{i}=\left(\tilde{S}, \tilde{T}^{0}, \tilde{H}_{d}^{0}, \tilde{H}_{u}^{0}\right)$. The four mass eigenvalues are dominantly given by the four independent mass parameters $\left(M_{B}^{D}, M_{W}^{D}, \mu_{d}, \mu_{u}\right)$, see appendix A. In contrast, in the MSSM the neutralinos are Majorana fermions, and there is only a single higgsino mass parameter $\mu$, so that two neutralino masses are approximately degenerate.

The MRSSM comprises four different charginos, with masses determined by the wino and the two higgsino mass parameters, see appendix A. In contrast, the MSSM contains only two charginos.

Table 3 gives an overview of possible observed signatures of sets of charginos and neutralinos (listed in the first column). It then shows the corresponding possible interpretations of the signatures in the MSSM (second column) and in the MRSSM (third column), which are very different as can be understood on the basis of the previous remarks. The table is more general than necessary for the purposes of the present paper, and it can serve as a basis of further investigations of the LHC phenomenology of the MRSSM.

\footnotetext{
${ }^{2}$ Similar limits are also given by the CMS collaboration [74].
} 
In general, as the number of degrees of freedom for neutralinos and charginos is twice as large in the MRSSM than in the MSSM one could naively expect an enhancement of the production cross section by a factor of four. However, all MRSSM charginos and neutralinos carry non-vanishing R-charge. Due to R-charge conservation, therefore, only half of all final state combinations is allowed. Furthermore, the new genuine MRSSM states $\left(\tilde{R}_{d}, \tilde{R}_{u}, \tilde{S}, \tilde{T}\right)$ do not interact at tree level with fermions, sfermions or gluons. Hence the situation is more complicated and has to be analyzed channel by channel.

Further differences between MRSSM and MSSM exist in the couplings of charginos and neutralinos and therefore in the decay branching ratios. For example, in the MSSM the higgsino mass parameter $\mu$ induces a maximal mixing between the up- and down-higgsino so that both will mix almost similarly with the bino and wino, implying an appreciable decay rates of both higgsinos to sleptons, if kinematically available. This is different in the MRSSM. R-symmetry does not allow a parameter which induces mixing between the up- and down-(R-)higgsinos, instead separate mass parameters $\mu_{d}$ and $\mu_{u}$ are needed. Then, up- and down-(R-)higgsino states separately will be good approximations to the corresponding mass eigenstates. For $\tan \beta \gg 1$ this also means that the mixing between down-(R-)higgsino with the wino-triplino and bino-singlino states will be strongly suppressed. As a result, for large $\tan \beta$ the down-(R-)higgsino has appreciable couplings only to staus, which are not shared with the up-(R-)higgsino.

It is also important to consider decays of charginos and neutralinos into the SM-like Higgs boson $H_{2}$ observed at the LHC or into the light singlet $H_{1}$, present in our scenario. These decays are influenced by the $\lambda / \Lambda$ parameters. Large $\Lambda$ are needed to generate large enough loop contributions to the mass of the SM-like Higgs boson and $\lambda$ needs to be small in the light singlet scenario studied here. Therefore, the decay of the wino-triplino to the SM-like Higgs boson and LSP, if kinematically allowed, will be enhanced by $\Lambda$, while contributions from $\lambda$ to the higgsinos decaying to the SM-like Higgs boson and LSP are small.

When the chargino/neutralino decay to the SM-like Higgs boson is kinematically not possible, decays to the light singlet in our scenario still might open. Therefore, it can be a competing channel to the decay to the $\mathrm{Z}$ boson above the $\mathrm{Z}$ boson mass or the dominant decay channel below it. For higher neutralino masses the branching ratio to the light singlet will be sub-dominant to the one to the SM-like Higgs boson, but might still be non-negligible, depending on the mixing between singlet and doublets.

\subsection{Setup for recasting LHC limits for the MRSSM}

After having given an overview of the qualitative details of electro-weak production in the MRSSM, we now turn to a more in-depth analysis, taking the experimental input into account in a systematic way.

In recent years different computational tools emerged, which try to automatize the study of beyond the Standard Model physics at the LHC in a rather generic way using standardized interfaces. This allows us to take the model differences between the MRSSM and the MSSM/simplified models studied by the experiments into account and directly 


\begin{tabular}{|c|c|c|}
\hline experimental signature & MSSM & MRSSM \\
\hline $\begin{array}{l}1 \text { charged and } \\
1 \text { neutral of similar mass }\end{array}$ & wino-like states & $\begin{array}{l}\text { either up- or } \\
\text { down-(R-)higgsino-like states }\end{array}$ \\
\hline $\begin{array}{l}2 \text { charged and } \\
1 \text { neutral of similar mass }\end{array}$ & - & wino-triplino-like states \\
\hline $\begin{array}{l}1 \text { charged and } \\
2 \text { neutral of similar mass }\end{array}$ & higgsino-like states & - \\
\hline $\begin{array}{l}2 \text { charged and } \\
2 \text { neutral of similar mass }\end{array}$ & - & $\begin{array}{l}\text { either up- or } \\
\text { down-(R-)higgsino-like states }\end{array}$ \\
\hline $\begin{array}{l}2 \text { charged and } \\
3 \text { neutral of similar mass }\end{array}$ & all states & (2) \\
\hline $\begin{array}{l}3 \text { charged and } \\
2 \text { neutral of similar mass }\end{array}$ & - & $\begin{array}{l}\text { wino-triplino-like and either up- or } \\
\text { down-(R-)higgsino-like states }\end{array}$ \\
\hline $\begin{array}{l}4 \text { charged and } \\
3 \text { neutral of similar mass }\end{array}$ & - & all states \\
\hline
\end{tabular}

Table 3. Possible discovery scenarios at colliders of different sets of particles from the neutralinochargino sector and the corresponding dominant gauge eigenstates for the MSSM and MRSSM. This is assuming the light-singlet-scenario in the MRSSM, which leads to a light bino-singlino as LSP candidate.

calculate bounds from the LHC on the MRSSM parameter space. In the following, we give a description of the set of tools used to for this.

Using the UFO [76] output produced by SARAH with Herwig++-2.7 $[77,78]^{3}$ we simulate the LO production of electro-weak sparticles at the LHC and compare it with $8 \mathrm{TeV}$ data with CheckMATE-1.2.0 [81]. ${ }^{4}$ CheckMATE includes ATLAS analyses, which can be sensitive to the final state of these processes, when several leptons appear as decay products. Specifically, we take into account the analyses implemented in CheckMATE for two [73, 87], three [88, 89] and four and more leptons [90] in the final state. The final output of CheckMATE used to set exclusion limits in the MRSSM parameter space is the value for $C L_{S}$ of most sensitive signal region of the studied analyses for each parameter point.

To ensure correctness of the calculated limits several tests of the used tools were done. For the calculation of the matrix element the event generator Herwig++-2.7 was checked against Madgraph 5 [91], FeynArts/FormCalc and CalcHEP [92], where also the input of the model files was produced by SARAH. Agreement was achieved up to the implementation differences of the programs. The analyses implementation by CheckMATE was checked by re-calculating some of the bounds given by experiment. Additionally, the cutflow for a selection of signal regions was reproduced by an independent implementation within uncertainties.

\footnotetext{
${ }^{3}$ Herwig++-2.7 is used with default settings, underlying event tune (UE-EE-5-MRST [79]) and PDF set (LO* from [80]).

${ }^{4}$ With CheckMATE we make use of Delphes 3 [82], FastJet [83], the anti- $k_{t}$ clustering [84, 85], and the $C L_{s}$ description [86].
} 


\begin{tabular}{|ccccccccc|}
\hline process & $\chi^{0} \bar{\chi}^{0}$ & $\chi^{0} \chi^{-}$ & $\chi^{0} \rho^{+}$ & $\chi^{+} \bar{\chi}^{0}$ & $\rho^{-} \bar{\chi}^{0}$ & $\chi^{+} \chi^{-}$ & $\rho^{+} \rho^{-}$ & $\tilde{\ell}_{R, i}^{+} \tilde{\ell}_{R, j}^{-}$ \\
\hline BMP4 & 496 & 619 & 3.9 & 1147 & 0.83 & 496 & 1.25 & 17.6 \\
BMP5 & 4.77 & 5.58 & 10.0 & 15.6 & 1.99 & 7.93 & 2.88 & $1.48 \times 10^{-3}$ \\
BMP6 & 1.67 & 6.04 & 22.6 & 17.0 & 5.65 & 12.1 & 8.48 & 38.4 \\
\hline
\end{tabular}

Table 4. EW cross sections as calculated by Herwig++ using the SARAH model input. All values given in $\mathrm{fb}$.

\begin{tabular}{|cccccccccccccc|}
\hline & $\chi_{1}^{0}$ & $\chi_{2}^{0}$ & $\chi_{3}^{0}$ & $\chi_{4}^{0}$ & $\chi_{1}^{ \pm}$ & $\chi_{2}^{ \pm}$ & $\rho_{1}^{ \pm}$ & $\rho_{2}^{ \pm}$ & $\tilde{\tau}_{R}$ & $\tilde{\mu}_{R}$ & $\tilde{e}_{R}$ & $\tilde{\ell}_{L}$ & $m_{H_{1}}$ \\
\hline BMP4 & 49.8 & 132 & 617 & 691 & 131 & 625 & 614 & 713 & 128 & 802 & 802 & 808 & 100 \\
BMP5 & 43.9 & 401 & 519 & 589 & 409 & 524 & 519 & 610 & 1000 & 1001 & 1001 & 1005 & 94 \\
BMP6 & 29.7 & 427 & 562 & 579 & 422 & 562 & 433 & 587 & 106 & 353 & 353 & 508 & 95 \\
\hline
\end{tabular}

Table 5. Masses of the non-SM particles in the BMPs relevant for the LHC studies discussed here. All values given in $\mathrm{GeV}$.

With our setup we will consider the following production processes $p p \rightarrow \chi^{0} \bar{\chi}^{0}, p p \rightarrow$ $\chi^{0} \chi^{+}, \chi^{-} \bar{\chi}^{0}, p p \rightarrow \chi^{0} \rho^{-}, \rho^{+} \bar{\chi}^{0}, p p \rightarrow \tilde{\ell}_{R}^{+} \tilde{\ell}_{R}^{-}$. The corresponding production cross sections of our benchmark points are given in table 4 . Processes with left-handed sleptons are neglected, assuming that the masses are above the detection limit as discussed before. The masses of the relevant particles are given in table 5 .

In the next sections we will show how the setup described here is used to recast the experimental bounds on the chargino and neutralino sector of the MRSSM. We will also highlight differences to the usual MSSM interpretations.

\subsection{Light staus and light charginos}

In the following we focus on specific cases, which are of interest for the light-singlet scenario and which are promising in view of dark matter. We begin with the case corresponding to the first row of table 3, i.e. we consider a very light LSP (a bino-like in the MSSM, and a bino-singlino in the MRSSM), and a relatively light chargino (a wino in the MSSM, and a down-(R-)higgsino in the MRSSM). We also take relatively light staus, which are motivated by dark matter considerations.

Figure 3 shows the resulting exclusion plots taking into account the experimental analyses as discussed in the last section. The results show striking differences between the MSSM and the MRSSM cases. In the MSSM the produced charginos are wino-like and all possible decay channels (Higgs, W/Z, staus) can contribute with comparable size to their total width. Therefore, as none of the signal regions of the analyses performed by the LHC experiments have been designed to accept all events/scenarios, the derived limits are rather weak. Especially for $m_{\chi_{2}^{0}}>m_{\chi_{1}^{0}}+m_{h}$ the decay to Higgs channel is dominant. The experimental analyses used here rely on final states with charged leptons and are not sensitive to Higgs bosons in the final state, yielding a low exclusion power. ${ }^{5}$ Only two small

\footnotetext{
${ }^{5}$ Dedicated analyses taking Higgs bosons in the final state into account exist but are not implemented in the used version of CheckMATE [74, 93].
} 

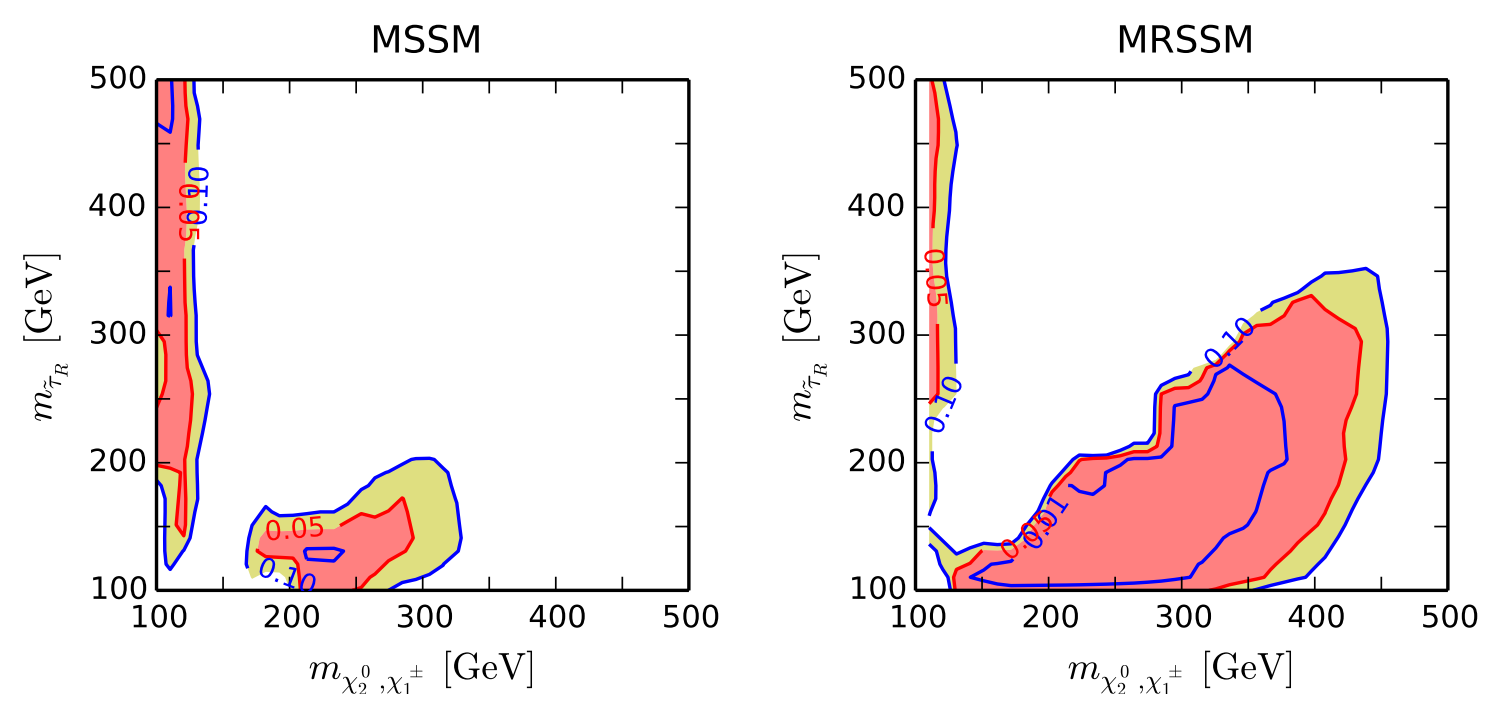

Figure 3. Exclusion plots in the chargino-neutralino and stau mass plane for the first scenario of table 3 in the MSSM (left) and in the MRSSM (right).

regions are excluded: the region with a chargino mass below 100-150 GeV (depending on the stau mass), and the region around the chargino mass of $150-300 \mathrm{GeV}$ and stau mass below $150 \mathrm{GeV}$. In contrast, for the MRSSM the produced charginos are down-higgsinolike and the decay to stau, if available, is preferred. This makes the searches designed for events with large multiplicity of taus very efficient. Hence, a large triangular region with chargino mass between the decay threshold $\left(m_{\tilde{\tau}_{R}}+m_{\mathrm{LSP}}\right)$ and around $450 \mathrm{GeV}$ is excluded. Interestingly, however, to the left of the triangle the chargino becomes too light, decays to on-shell staus are impossible, and decays via off-shell stau are suppressed. In that region the acceptance is lowered and with present Run-I data no exclusion is possible.

\subsection{General investigation of light charginos}

Now we discuss the limits on chargino and neutralino masses in more generality. Fixing the bino-singlino to be very light, $\sim 50 \mathrm{GeV}$, there are three relevant mass parameters: the Dirac wino-triplino mass $M_{W}^{D}$ and the two higgsino masses $\mu_{d, u}$. Further, the exclusion limits depend on the slepton masses.

Figure 4 shows the exclusion regions in the plane of the two higgsino masses $\mu_{d}-\mu_{u}$, for the case that also right-handed staus are light. In the left plot all other sleptons are heavy; in the right plot the right-handed sleptons of the other generations are also light. In both plots there is an interesting non-excluded region with very small $\mu_{d}$, below around $150 \mathrm{GeV}$. This is consistent with the discussion of the previous subsection and with the corresponding region in figure 3 . The region for $\mu_{d}$ between around 150 and $400 \mathrm{GeV}$ corresponds to the triangular region in figure 3, in which the decay of the higgsino into stau is dominant, and is excluded. In the case of light right-handed sleptons of all generations, the region for the other higgsino mass $\mu_{u}$ below around $300 \mathrm{GeV}$ is also excluded. This originates from the mixing with the bino, leading to non-negligible and democratically distributed decay to all right-handed sleptons in addition to the decay to $\mathrm{Z}$ and Higgs boson. Therefore 

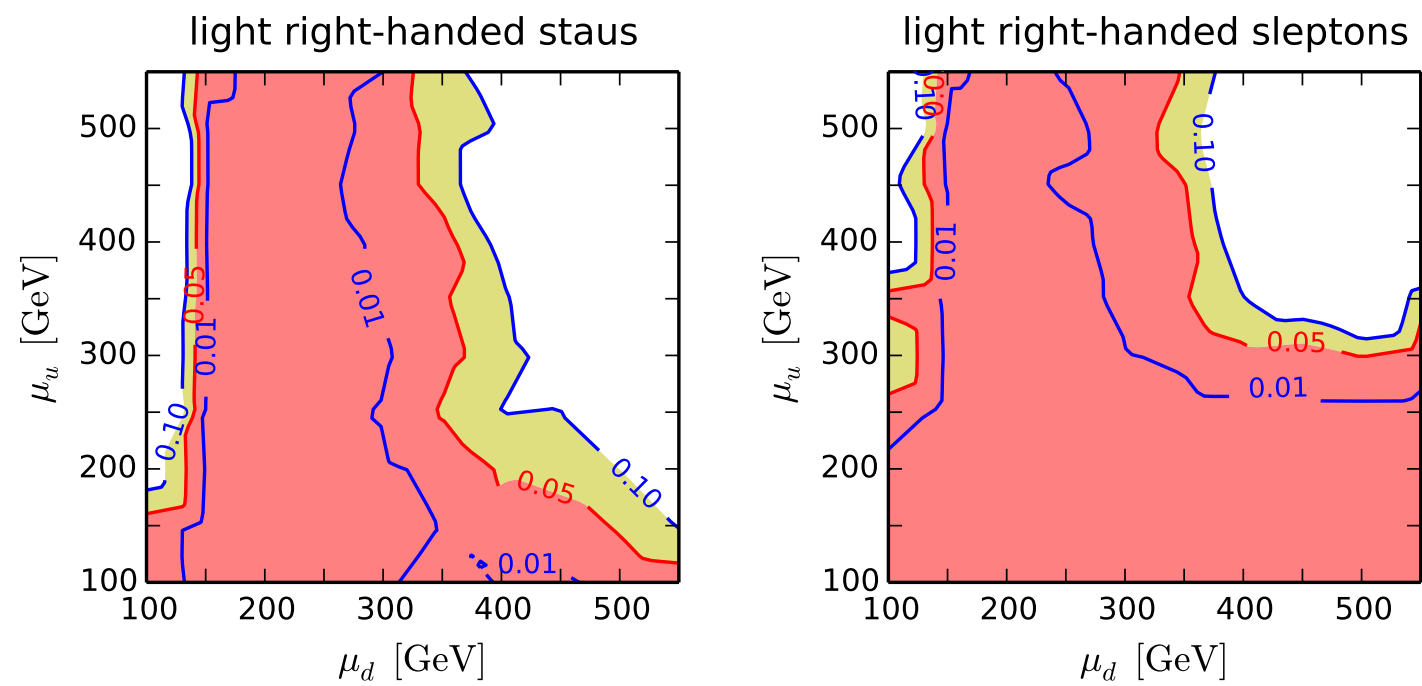

Figure 4. Exclusion limits in the MRSSM for light staus as a function of the two higgsino mass parameters $\mu_{d, u}$. The red (yellow) region marks the 95\% (90\%) excluded parameter region, computed as described in the text. The left plot has light right-handed stau mass of $100 \mathrm{GeV}$, in the right plot all right-handed slepton masses are set to $100 \mathrm{GeV}$. All other parameters are fixed to the values of BMP5. Most importantly, the Dirac wino mass is set to $M_{W}^{D}=500 \mathrm{GeV}$.

selectrons and smuons will be produced in large enough abundance to be picked up by the experiments. As discussed before, this is in contrast to the down-higgsino, where the mixing to the bino is suppressed via large $\tan \beta$ and the large Yukawa coupling of tau leads to dominating decay into staus. In the case of heavy sleptons of the first two generations, the exclusion power on $\mu_{u}$ is very weak as the decay to $\mathrm{Z}$ and Higgs boson have comparable branching ratios. Thus the case of light right-handed staus leads to two distinct viable parameter regions, one with very small $\mu_{d}$ and one with larger $\mu_{d}$. These two regions are represented by the two benchmark points BMP4 and BMP6.

Now we consider the case in which all sleptons, including the staus, are heavy. Figure 5 shows the exclusion regions for this case, once in the $\mu_{d}-\mu_{u}$ plane (with $M_{W}^{D}=500 \mathrm{GeV}$ ), and once in the $\mu_{d}=\mu_{u}-M_{W}^{D}$ plane. Both plots show that once both higgsinos are heavier than around $300 \mathrm{GeV}$ there are parameter regions that are safe from exclusion. This generic, viable parameter region for heavy sleptons is represented by benchmark point BMP5.

The left plot of figure 5 analyzes the case that one of the higgsinos is lighter. It shows two additional strips of parameter space at higgsino masses around $150 \mathrm{GeV}$, which are not excluded by the considered LHC searches. The physics reason for the existence of these regions are the higgsino decay patterns. In the non-excluded strips the dominant decay of the light higgsino is a two-body decay into the LSP and W/Z or SM-like Higgs boson. Due to the small higgsino mass, the LSP is too soft to allow a discrimination of the events from the background of standard on-shell W/Z/Higgs production and decay. For even lower higgsino masses, the dominant decay modes are 3-body decays via the SM bosons. In this case the SM background for the lepton searches is smaller and the exclusion power higher. We exemplify the region of low $\mu_{d}$ by the benchmark point BMP4. For the region of low 

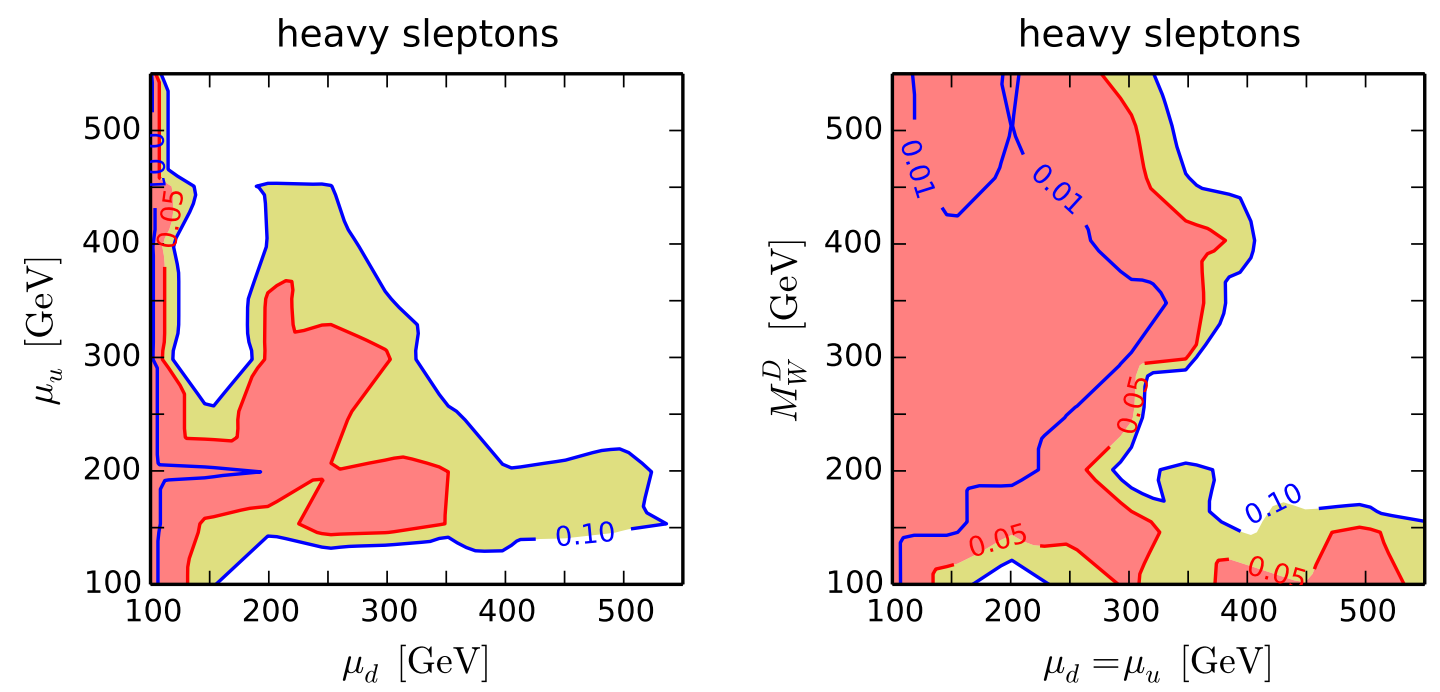

Figure 5. Exclusion limits in the MRSSM for heavy sleptons as a function of the two higgsino masses (left) and of the higgsino and wino-triplino masses (right). The red (yellow) region marks the $95 \%(90 \%)$ excluded parameter region. All parameters which are not varied in the plots are fixed to the values of BMP5.

$\mu_{u}$ we also need to consider constraints from dark matter searches, which will be described in the next section.

In the right of figure 5 masses of the wino-triplino $M_{W}^{D}$ below $200 \mathrm{GeV}$ for higgsino masses above $300 \mathrm{GeV}$ can not be excluded by the analyses used here. As was discussed before, the wino-triplino decays predominantly into Higgs bosons and LSP and the Higgs boson again mainly into bottom quarks. As described before, this gives a low exclusion power since the experimental analyses so far are not sensitive to the Higgs bosons in the final state.

In summary, our investigations show that there are three qualitatively different viable parameter regions compatible with very light bino-singlino state, represented by the three benchmark points BMP4,5,6. Two regions are characterized by light right-handed stau and either light or heavy down-higgsino (where light/heavy means masses around $100 \mathrm{GeV}$ /larger than around $400 \mathrm{GeV}$, respectively). The third region is characterized by generally heavy sleptons and higgsino masses above around $300 \mathrm{GeV}$. In all cases, the winotriplino mass is not very critical; the benchmark points have $M_{W}^{D}$ between $400-600 \mathrm{GeV}$, but smaller values are allowed as well, and do not give rise to a different phenomenology.

\section{Dark matter constraints}

As was pointed out in section 2 the scenario with a light singlet Higgs state considered in this work leads to an upper bound on the bino-singlino mass parameter $M_{B}^{D}<55 \mathrm{GeV}$. The neutralino, which is mainly given by this component, will become the LSP. Therefore, it is the dark matter candidate of our model and we will study here how viable this scenario is when confronted with constraints from dark matter searches. Dirac neutralinos as a candidate for dark matter were already studied in ref. [34, 36]. Especially in ref. [36] 
it was clarified that the bino-singlino state is viable candidate as it is possible to achieve the correct relic abundance by annihilation to leptons through sleptons, especially a light right-handed stau. Additionally, the spin-independent direct detection channels were investigated and the squark and $\mathrm{Z}$ boson exchange as main contributions were identified. Interference between those two channels was neglected, but we will see in the following that it plays an important role to evade the direct detection limits.

The technical setup for the numerical computations described below is as follows. We use MicrOMEGAs-4.1.8 [94] to calculate the relic density and direct detection rate for the MRSSM. The model input is given by the CalcHEP output of SARAH and for each parameter point the mass spectrum and couplings calculated with SPheno at full one-loop level is passed to the program. For the comparison to the experimental data and statistical interpretation of the direct detection part we use LUXCalc [95], which uses results from the LUX experiment [96] and the cross section for each parameter point from MicrOMEGAs.

\subsection{Relic density}

We first consider the question which parameter space of the light-singlet scenario is compatible with the measured dark matter relic density. As in the MSSM, the crucial requirement is to achieve sufficiently effective LSP pair annihilation processes. In the parameter regions found in the previous sections, it turns out that two cases are promising. Either, if the condition $m_{\chi_{1}} \approx M_{Z} / 2$ is valid and s-channel resonant LSP pair annihilation into Z bosons is possible, or if right-handed staus are light and enable annihilation via t-channel stau exchange into tau leptons, see the corresponding Feynman diagrams in figure 6 , when $f, \tilde{f}$ replaced by $\tau, \tilde{\tau}$ for non-Z-exchange diagrams.

Figure 7 (left) shows the resulting allowed contour in the $M_{B}^{D}-m_{\tilde{\tau}_{R}}$ parameter space. In the plot, all non-varied parameters are fixed to the values of BMP6, but this choice is not crucial. The important feature is that, as in the MSSM, to meet the measured value of the relic density the stau mass has to lie in a small interval once the other parameters are given. The contour has a sharp resonance-like peak around $M_{B}^{D} \approx M_{Z} / 2$ which results from the s-channel annihilation process. The two different possibilities mentioned above correspond to being at the resonance or away from it.

In the resonance peak the required stau mass has to be rather high. This is the situation realized in our benchmark point BMP5. For this scenario to be viable it has to be ensured that the $\mathrm{Z}$ boson does not decay to the LSP with a detectable effect to the invisible width of the $\mathrm{Z}$ boson. Since the same interaction is also important for, and thus constrained by, the direct detection cross section, we will discuss it in the subsequent subsection.

For values of $M_{B}^{D}$ away from the resonance, figure 7 (left) shows that the required stau mass is small and below $150 \mathrm{GeV}$. The benchmark points BMP4 and BMP6 represent this case. For such light staus the LEP bound on the stau mass of $\approx 82 \mathrm{GeV}$ [97] needs to be taken into account. In general, the LEP bound implies that there is a lower limit on $M_{B}^{D}$ for which the dark matter density can be explained.

Table 6 provides the precise values for the LSP and right-handed stau masses, as well as the relic density, for the benchmark points. Again the different characteristics of BMP5 and BMP4/BMP6 are visible. The benchmark point BMP4 has been tuned to give exactly 


\begin{tabular}{|cccc|}
\hline & BMP4 & BMP5 & BMP6 \\
\hline$m_{\chi^{1}}$ & $49.8 \mathrm{GeV}$ & $43.9 \mathrm{GeV}$ & $29.7 \mathrm{GeV}$ \\
$m_{\tilde{\tau}_{R}}$ & $128.5 \mathrm{GeV}$ & $1 \mathrm{TeV}$ & $105.6 \mathrm{GeV}$ \\
$\Omega h^{2}$ & 0.119 & 0.092 & 0.127 \\
direct detection p-value & 0.9 & 0.5 & 0.2 \\
\hline
\end{tabular}

Table 6. Values of dark matter observables for the benchmark points.
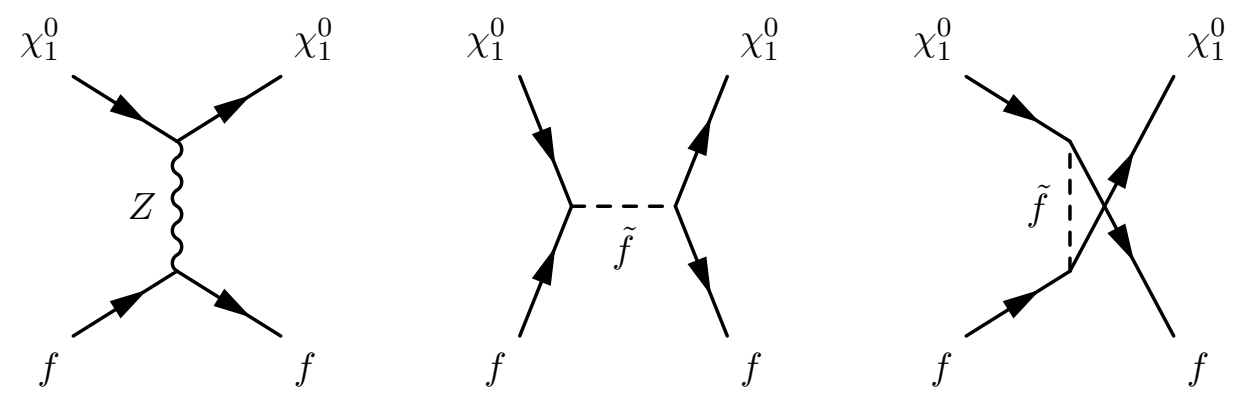

Figure 6. Feynman diagrams for the most relevant LSP annihilation (with $f, \tilde{f}$ replaced by $\tau, \tilde{\tau}$ ) and direct detection processes $(f, \tilde{f}$ replaced by $u, \tilde{u}$ and $d, \tilde{d})$.

the experimental value of $\Omega h^{2}=0.1199 \pm 0.0027$ [98]. For BMP5 (BMP6) the bino-singlino (stau) mass can easily be tuned by a small percentage to achieve full agreement with the measured $\Omega h^{2}$.

\subsection{Direct detection}

The spin-independent dark matter-nucleon scattering cross section can be given in terms of two scattering amplitudes $f_{p}, f_{n}$. These are conventionally normalized such that the total spin-independent cross section at zero momentum transfer is

$$
\sigma_{D M-N}=\frac{4 \mu_{Z_{A}}^{2}}{\pi}\left(Z f_{p}+(A-Z) f_{n}\right)^{2} .
$$

Here $\mu_{Z_{A}}^{2}$ is the dark matter-nucleon reduced mass, and $A$ and $Z$ are atomic mass and number, respectively.

As noted in ref. [36], the spin-independent cross-section for Dirac neutralinos is dominated by the vector part of the $\mathrm{Z}$ boson-exchange and squark-exchange contributions. The relevant Feynman diagrams are shown in figure 6 , when $f, \tilde{f}$ replaced by $u, \tilde{u}$ and $d, \tilde{d}$. Each contribution can lead to large scattering rates and thus to strong bounds on the parameter space. For this reason we provide explicit expressions for the relevant amplitudes. For the Z-mediated diagram we obtain

$$
\begin{aligned}
f_{p}^{Z \text { boson }} & =\frac{-g_{2}^{2}}{8 m_{Z}^{2} c_{W}^{2}}\left(\frac{1}{2}-2 s_{W}^{2}\right)\left(\frac{Z_{1,3-4}+Z_{2,3-4}}{2}\right), \\
f_{n}^{Z \text { boson }} & =\frac{g_{2}^{2}}{16 m_{Z}^{2} c_{W}^{2}}\left(\frac{Z_{1,3-4}+Z_{2,3-4}}{2}\right),
\end{aligned}
$$


where $Z_{i, 3-4}=\left(N_{13}^{i}\right)^{2}-\left(N_{14}^{i}\right)^{2}$ is the difference of the mixing matrix elements squared for the mixing of the bino with the down- and up-R-higgsino (singlino with the down- and up-higgsino) when $i=1(i=2)$, see eqs. (A.2) and (A.3). Here we also use the shorthand notation $s_{W}=\sin \theta_{W}, c_{W}=\cos \theta_{W}$.

The coupling factors $Z_{i, 3-4}$ arise because the $\mathrm{Z}$ boson only couples to the (R-)higgsino content of the LSP. Qualitatively, in order to suppress these amplitudes and to ensure agreement with current direct detection bounds, the (R-)higgsino mix-in to the LSP has to be suppressed. This leads particularly to constraints on the higgsino mass parameters $\mu_{u}$ and $\mu_{d}$. As a simple illustration, we assume $\tan \beta \gg 1, \lambda_{u}=0$, and $\mu_{u} \gg M_{B}^{D}, g_{1} v$ and further that the direct mixing between up-higgsino and bino will be dominant. In this case, the mixing matrix elements appearing in eqs. (4.2) and (4.3) take the form

$$
Z_{1,3-4}+Z_{2,3-4}=-\left(\frac{g_{1} v}{2 \mu_{u}}\right)^{2}
$$

clearly exhibiting the suppression by large $\mu_{u}$. Similarly, we have evaluated the squarkmediated diagrams of figure 6 for general masses of the four first-generation squarks, $\tilde{u}_{L}$, $\tilde{u}_{R}, \tilde{d}_{L}, \tilde{d}_{R}$. For the qualitative discussion it is sufficient to provide the results in the limit of heavy squarks of equal mass $m_{\tilde{q}}=m_{\tilde{d}} \approx m_{\tilde{u}} \gg m_{\chi_{1}}$. In this limit we obtain

$$
\begin{aligned}
f_{p}^{\text {heavy squark }} & =\frac{g_{1}^{2}}{4 m_{\tilde{q}}^{2}}\left[\left(\frac{4}{9}-\frac{1}{36}\right)+\frac{1}{2}\left(\frac{1}{9}-\frac{1}{36}\right)\right], \\
f_{n}^{\text {heavy squark }} & =\frac{g_{1}^{2}}{4 m_{\tilde{q}}^{2}}\left[\frac{1}{2}\left(\frac{4}{9}-\frac{1}{36}\right)+\left(\frac{1}{9}-\frac{1}{36}\right)\right] .
\end{aligned}
$$

The minus signs result from the different Dirac structure of the $\mathrm{s}$ and $\mathrm{u}$ channels that contribute to the process. Therefore, in order to suppress these amplitudes and ensure agreement with current bounds, the squarks need to be sufficiently heavy. ${ }^{6}$

For our quantitative evaluation we include the full experimental information from LUX by using LUXCalc and the complete theoretical prediction of the proton and neutron scattering amplitudes. A likelihood is then constructed from Poisson distribution

$$
\mathcal{L}\left(m_{\chi},\left\{f_{N}\right\} \mid N\right)=\frac{(\mu+b)^{N} e^{-(\mu+b)}}{N !},
$$

where $N$ and $b$ are the observed and expected numbers of events by the LUX experiment, respectively, and $\mu$ is the expected number of signal events for a given WIMP mass and its effective couplings. Furthermore, we assume that DM consists in equal proportions from $\chi$ and $\bar{\chi}$ and use default settings for the halo profile in MicrOMEGAs.

On the right of figure 7, the $95 \%$ and $90 \%$ exclusion bounds (violet (dark) and yellow (light dark) regions) derived using the log likelihood for the direct detection by LUX given in eq. (4.7) are shown depending on $\mu_{u}$ and the first/second generation squark masses.

\footnotetext{
${ }^{6}$ Our expressions for the direct detection amplitudes differ by a factor $1 / 16$ in eqs. (4.2), (4.3) and by the relative sign between the left-handed and right-handed squark contributions in eqs. (4.5), (4.6) from the corresponding ones of ref. [36].
} 
Here, it can be seen that the derived limits are quite sensitive to the combination of both parameters. This dependence stems from an interference of the amplitudes and can be understood by a simplified analysis of the expressions, as given before.

In the approximation leading to eq. (4.4), we can add the Z- and squark-exchange contributions to a simple form

$$
f_{n}^{Z} \text { boson+heavy squark }=\frac{g_{1}^{2}}{96}\left(\frac{7}{m_{\tilde{q}}^{2}}-\frac{3}{\mu_{u}^{2}}\right), \quad f_{p}^{Z \text { boson+heavy squark }}=\frac{g_{1}^{2}}{96}\left(\frac{11}{m_{\tilde{q}}^{2}}\right),
$$

where $s_{W}^{2}=1 / 4$ has been taken in the proton case. Plugging these results into the expression for the dark matter-nucleus cross section, eq. (4.1), we find a complete destructive interference, i.e. $\sigma_{D M-N}=0$, when

$$
m_{\tilde{q}}=\sqrt{\frac{7+11 \frac{Z}{A-Z}}{3}} \mu_{u} \underset{\text { Xe }}{\approx} 2.2 \mu_{u},
$$

where the numerical value for xenon is calculated with $A=131.3$ and $Z=54$.

The line of destructive interference corresponding to eq. (4.9), shown as a full line in figure 7 (right), explains the funnel-shaped allowed region. Above the line, the squarkmediated amplitudes become small, to the right of the line, the Z-mediated amplitudes become small. It should be noted that the result for the exclusion bounds is calculated using the complete information of micrOMEGAs and LUXCalc, while eq. (4.9) is only approximated. As the squark masses of the first two generations are limited by LHC searches, the direct detection non-discovery provides a lower limit on $\mu_{u}$. This also excludes the region of low $\mu_{u}$ allowed by LHC searches in the left of figure 5 .

As discussed before, the amplitude for the Z-mediated exchange is closely related to the one responsible for the $\mathrm{Z}$ boson decay to a pair of LSP. Both depend strongly on the $Z_{i, 3-4}$ containing the neutralino mixing matrix elements. The direct detection limit of figure 7 limits the $Z_{i, 3-4}$ strongly enough so that $\Gamma\left(Z \rightarrow \chi_{1}^{0} \bar{\chi}_{1}^{0}\right) \ll 3 \mathrm{MeV}$ is ensured over all the parameter space.

Xenon is not the only element used for direct detection experiments, therefore the change of the values of $A$ and $Z$ has to be taken into account, when using eq. (4.9) for other cases. The variation of the ratio $Z /(A-Z)$ ( 0.7 for xenon, 0.8 for argon and germanium) is small. Therefore, the bounds from different experiments will not differ much from each other. Here, we only take the LUX result into account as it is the most sensitive one available.

\section{Summary and conclusions}

The minimal R-symmetric model MRSSM is a promising alternative to the MSSM, which predicts Dirac gauginos and higgsinos as well as scalars in the adjoint representation of $\mathrm{SU}(3) \times \mathrm{SU}(2) \times \mathrm{U}(1)$. Here we have investigated the possibility that the scalar singlet mass is small and gives rise to a singlet-like mass eigenstate with mass below $125 \mathrm{GeV}$. The potential advantages of this scenario are an increased tree-level SM-like Higgs boson mass, many light weakly interacting particles which could be discovered at the next LHC run, and the possibility to explain dark matter. 

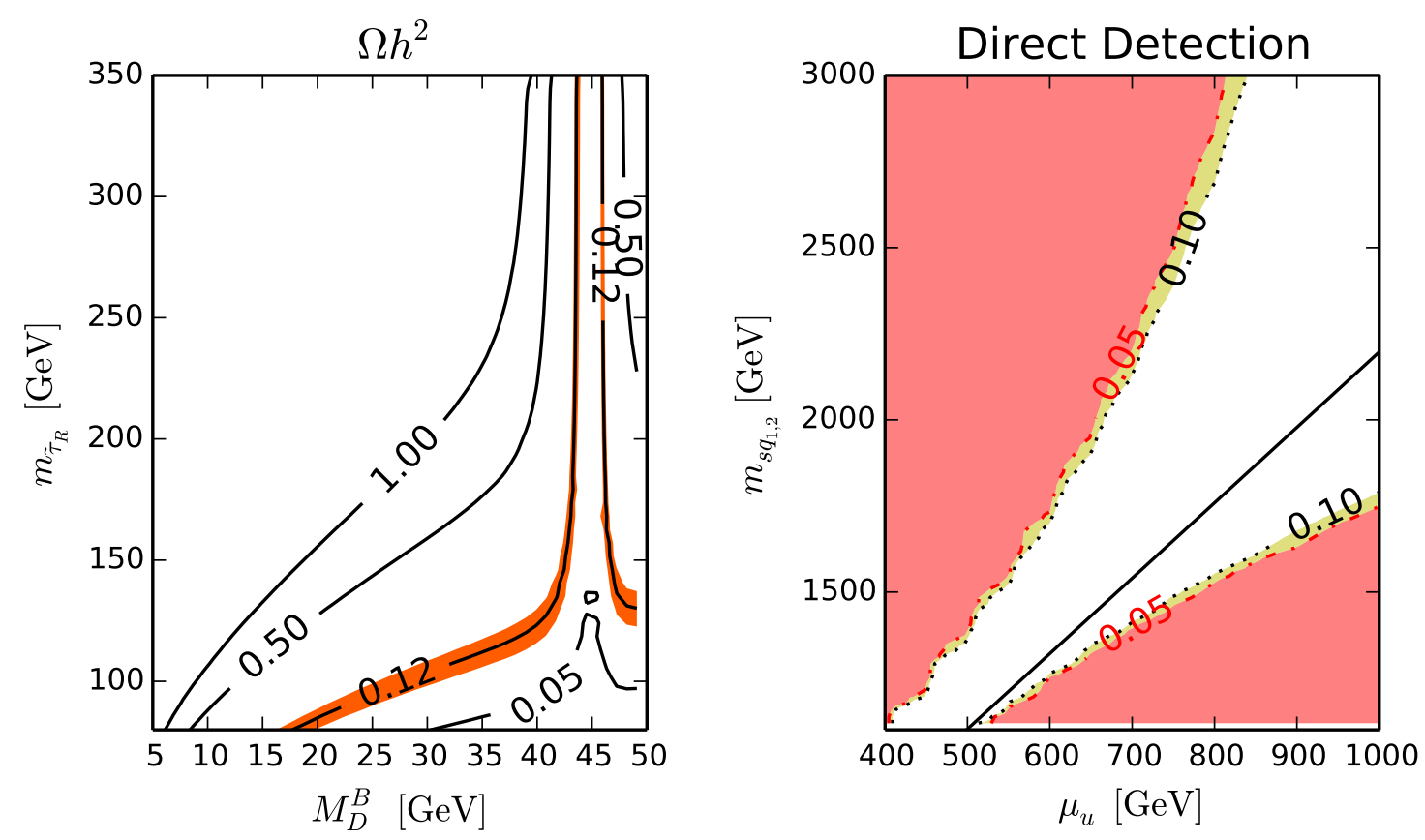

Figure 7. Dark matter relic density as a function of the bino mass parameter and right-handed slepton masses (left). Exclusion limits in the MRSSM depending on $\mu_{u}$ and equal first and second generation squark masses (right) from direct detection. The red with dashed dotted border (yellow with dotted border) region marks the 95\% (90\%) excluded parameter region calculated with micrOMEGAs and LUXcalc. The full line shows the approximation relation for the full destructive interference in eq. (4.9). The non-varied parameters in each plot are fixed to the values of BMP6.

Many of the model parameters are strongly constrained in order to make this scenario viable. Here we briefly summarize the main constraints. Figure 8 summarizes the four most important non-LHC constraints on the relevant parameters. There, the SM-like Higgs boson mass is given by the green-yellow colour bands, while the red area shows the regions excluded by direct detection searches. Black full (blue dashed) lines give the $\mathrm{W}$ boson mass $\left(\Omega h^{2}\right)$ contours. All plots are based on BMP6, except for figure 8(d), where BMP4 was used.

In order to realize a light singlet-like Higgs boson in the first place, not only the parameter $m_{S}$ but also $M_{B}^{D}$ and $\lambda_{u}$ must be very small. The SM-like Higgs boson mass is increased at tree-level compared to the case with heavy singlet or to the MSSM, but loop contributions governed by $\Lambda_{u}$ are still important. Accordingly, the plots of figure 8 show that the Higgs mass measurement essentially fixes $\Lambda_{u}$ and constrains $M_{B}^{D}$ and $\mu_{u}$. We have found that the other constraints in Higgs physics, arising from measurements of Higgs properties and of non-discovery of further Higgs states, can be easily fulfilled. As discussed in ref. [46], the W-boson mass can be explained simultaneously with the SM-like Higgs boson mass. Figure 8 confirms that this is still the case in the light singlet scenario.

The dark matter relic density can be explained in the MRSSM light singlet scenario because the Dirac bino-singlino neutralino is necessarily the stable LSP. Agreement with the observed relic density requires particular combinations of the bino-singlino mass $M_{B}^{D}$ and the right-handed stau mass. If the latter is heavy, as in our benchmark point BMP5, 


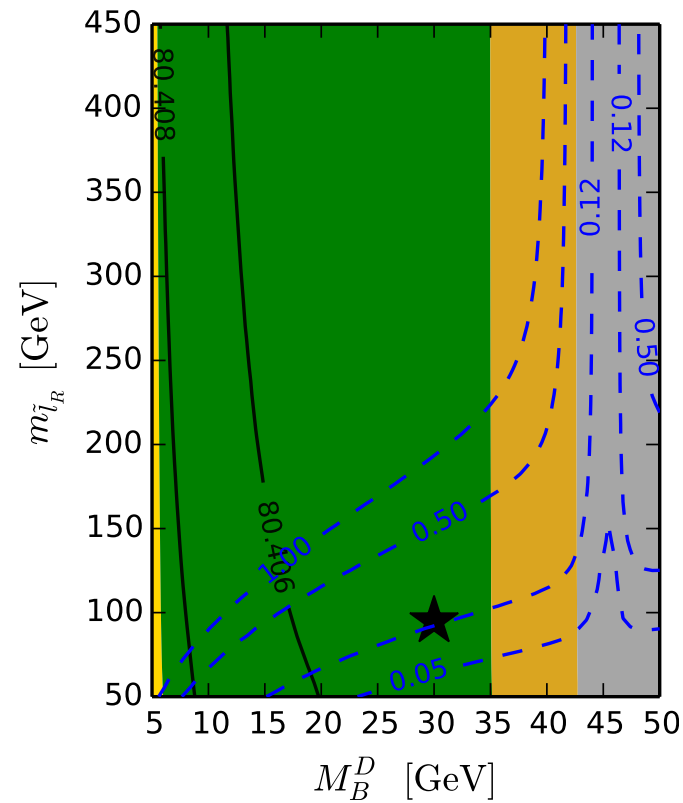

(a)

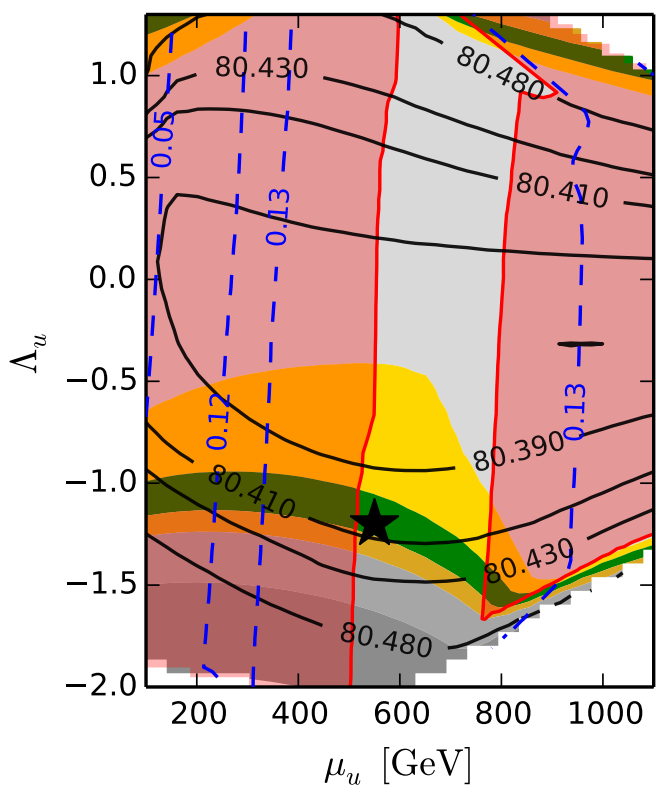

(c)

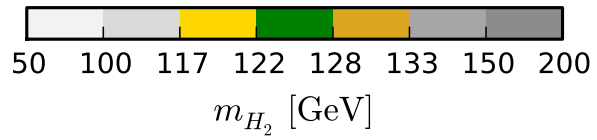

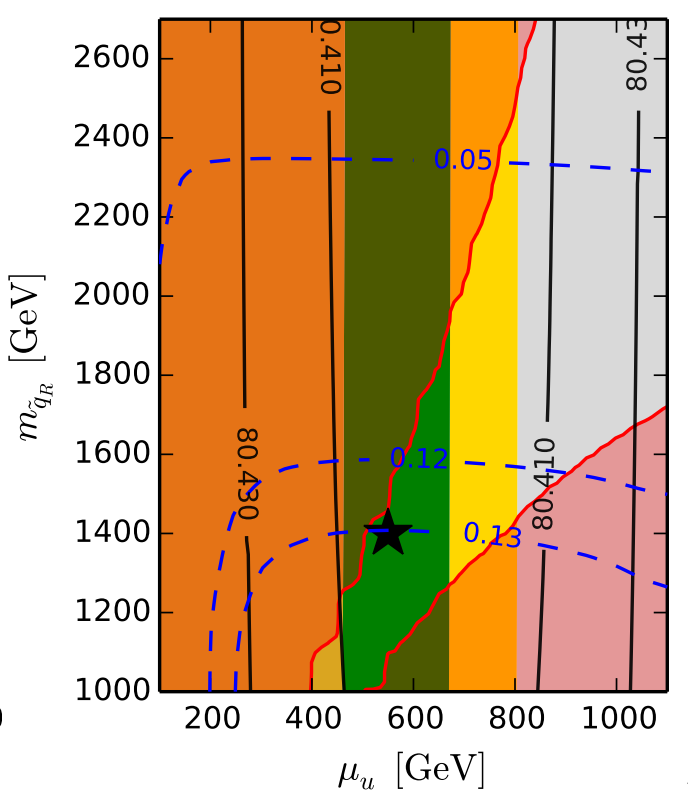

(b)

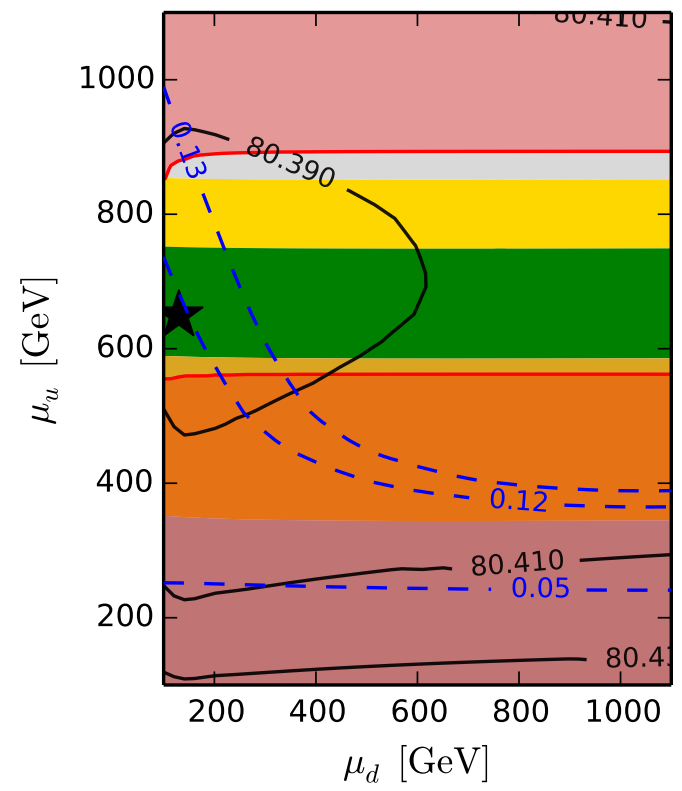

(d)

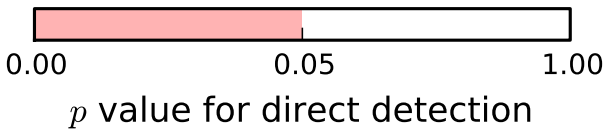

Figure 8. Shown are parameter regions and their agreement with experiment. Red overlaid areas are excluded with 95\% CL by dark matter direct detection. Black full (blue dashed) lines show $m_{W}$ $\left(\Omega h^{2}\right)$. The mass of the SM-like Higgs boson $m_{H_{2}}$ is given by the colour scale shown. All non-varied parameters are set to the values of BMP6, except for the bottom right plot, where BMP4 is used. BMPs are marked by stars. The white areas in (d) stem from tachyonic states appearing in the mass spectrum, at the borders to these areas the interpolation breaks down. 
the LSP mass must be close to half of the $\mathrm{Z}$ boson mass. If the LSP mass is away from the $\mathrm{Z}$ boson resonance, the right-handed stau must be light, as in our BMP4 and BMP6. This resonance behaviour is clearly displayed in figure $8(\mathrm{a})$. Parameters like $\mu_{u}, \mu_{d}$ influence the relic density due to their impact on the LSP mass via mixing, figure $8(\mathrm{~d})$.

Further bounds on the model parameters arise from the negative searches for dark matter and from the negative LHC searches for SUSY particles. The direct dark matter searches correlate $\mu_{u}$ with $m_{\tilde{q}_{R} ; 1,2}^{2}$, figure $8(\mathrm{~b})$. Because of the non-discovery of squarks at LHC so far, this gives rise to a lower bound on $\mu_{u}$, which is not affected by other parameters of the weak sector like $\Lambda_{u}$, figure 8(c). Together with the Higgs mass value, however, these searches constrain both $\mu_{u}$ and $m_{\tilde{q}_{R} ; 1,2}^{2}$ to a rather narrow range, see figure $8(\mathrm{~b})$.

Our recast of LHC analyses has revealed three distinct viable parameter regions of interest, represented by the three benchmark parameter points BMP4, BMP5, BMP6. The most obvious region is characterized by heavy $M_{W}^{D}, \mu_{d}$ and sleptons (BMP5). A second is characterized by very light right-handed stau mass around $100 \mathrm{GeV}$ (BMP6); in the third region the right-handed stau and $\mu_{d}$ are very light (BMP4). Figure 8(d), which gives the parameter variation for BMP4, shows that this region is also allowed by the other constraints. As explained in section 3, these two regions are allowed because the existing LHC searches become ineffective. The LHC searches alone would allow further parameter regions with very small $\mu_{u}$, which however are excluded by dark matter constraints. We note in passing that the parameter regions allowed by LHC and dark matter limits are valid also without a light singlet state.

To summarize: the experimental data from collider and dark matter experiments impose stringent constraints on the parameter of the light scalar scenario of the MRSSM. Nevertheless we have identified regions in the parameter space and proposed representative benchmark points BMP4,5,6 fulfilling all the constraints. All viable parameter regions are characterized by several light weakly interacting SUSY particles and will be tested both by future dark matter and LHC SUSY searches.

In all regions, the LSP will be the Dirac bino-singlino neutralino with a mass below $60 \mathrm{GeV}$. In two regions the right-handed stau is light and the down-higgsino either light (BM6) or heavy (BM4) (where light/heavy means masses around $100 \mathrm{GeV} /$ larger than around $400 \mathrm{GeV}$, respectively). The third region is characterized by generally heavy sleptons and higgsino masses above around $300 \mathrm{GeV}$ (BM5). The dark matter direct detection limit puts a lower bound on the up-type higgsino mass of around $500 \mathrm{GeV}$.

All these constraints leave a light stau, the wino-triplino and the down-type higgsino still in reach for the next LHC run. Evidence for the light singlet scenario would be the direct observation of a scalar state with a lower mass and one or more of the light states mentioned above.

\section{Acknowledgments}

Work supported in part by the German DFG Research Training Group 1504 and the DFG grant STO 876/4-1, the Polish National Science Centre grants under OPUS2012/05/B/ST2/03306 and the European Commission through the contract PITN-GA2012-316704 (HIGGSTOOLS). 
WK would like to thank Aleksandra Drozd for useful discussions concerning dark matter. PD would like to thank Daniel Schmeier for technical support with CheckMATE. We thank Christopher Savage for help on LuXCalc usage in the case of Dirac dark matter candidate.

\section{A MRSSM mass matrices at tree level}

For completeness we recall here the tree-level mass matrices for the Higgs bosons and the charginos and neutralinos of the MRSSM, which are relevant for the discussion of the present paper.

a) Pseudo-scalar Higgs bosons.

Since in the pseudo-scalar sector of $\left(\sigma_{d}, \sigma_{u}, \sigma_{S}, \sigma_{T}\right)$ there is no mixing between the MSSM-like states $\left(\sigma_{d}, \sigma_{u}\right)$ and the singlet-triplet states $\left(\sigma_{S}, \sigma_{T}\right)$, the mass-squared matrix breaks into two $2 \times 2$ submatrices as follows

$$
\mathcal{M}_{u, d}^{\sigma}=\left(\begin{array}{cc}
B_{\mu} \frac{v_{u}}{v_{d}} & B_{\mu} \\
B_{\mu} & B_{\mu} \frac{v_{d}}{v_{u}}
\end{array}\right), \quad \mathcal{M}_{S, T}^{\sigma}=\left(\begin{array}{cc}
m_{S}^{2}+\frac{\lambda_{d}^{2} v_{d}^{2}+\lambda_{u}^{2} v_{u}^{2}}{2} & \frac{\lambda_{d} \Lambda_{d} v_{d}^{2}-\lambda_{u} \Lambda_{u} v_{u}^{2}}{2 \sqrt{2}} \\
\frac{\lambda_{d} \Lambda_{d} v_{d}^{2}-\lambda_{u} \Lambda_{u} v_{u}^{2}}{2 \sqrt{2}} & m_{T}^{2}+\frac{\Lambda_{d}^{2} v_{d}^{2}+\Lambda_{u}^{2} v_{u}^{2}}{4}
\end{array}\right)
$$

b) Neutralinos.

In the weak basis of eight neutral electroweak two-component fermions: $\xi_{i}=\left(\tilde{B}, \tilde{W}^{0}\right.$, $\left.\tilde{R}_{d}^{0}, \tilde{R}_{u}^{0}\right), \zeta_{i}=\left(\tilde{S}, \tilde{T}^{0}, \tilde{H}_{d}^{0}, \tilde{H}_{u}^{0}\right)$ with R-charges $+1,-1$ respectively, the neutralino mass matrix takes the form of

$$
m_{\chi}=\left(\begin{array}{cccc}
M_{B}^{D} & 0 & -\frac{1}{2} g_{1} v_{d} & \frac{1}{2} g_{1} v_{u} \\
0 & M_{W}^{D} & \frac{1}{2} g_{2} v_{d} & -\frac{1}{2} g_{2} v_{u} \\
-\frac{1}{\sqrt{2}} \lambda_{d} v_{d} & -\frac{1}{2} \Lambda_{d} v_{d} & -\mu_{d}^{\text {eff, },} & 0 \\
\frac{1}{\sqrt{2}} \lambda_{u} v_{u} & -\frac{1}{2} \Lambda_{u} v_{u} & 0 & \mu_{u}^{\text {eff, },-}
\end{array}\right) .
$$

The transformation to a diagonal mass matrix and mass eigenstates $\kappa_{i}$ and $\psi_{i}$ is performed by two unitary mixing matrices $N^{1}$ and $N^{2}$ as

$$
N^{1, *} m_{\chi} N^{2, \dagger}=m_{\chi}^{\text {diag }}, \quad \xi_{i}=\sum_{j=1}^{4} N_{j i}^{1, *} \kappa_{j}, \quad \zeta_{i}=\sum_{j=1}^{4} N_{i j}^{2, *} \psi_{j},
$$

and physical four-component Dirac neutralinos are constructed as

$$
\chi_{i}^{0}=\left(\begin{array}{c}
\kappa_{i} \\
\psi_{i}^{*}
\end{array}\right) \quad i=1,2,3,4
$$

c) Charginos.

The mass matrix of charginos in the weak basis of eight charged two-component fermions breaks into two $2 \mathrm{x} 2$ submatrices. The first, in the basis $\left(\tilde{T}^{-}, \tilde{H}_{d}^{-}\right),\left(\tilde{W}^{+}, \tilde{R}_{d}^{+}\right)$ of spinors with R-charge equal to electric charge, takes the form of

$$
m_{\chi^{+}}=\left(\begin{array}{cc}
g_{2} v_{T}+M_{W}^{D} & \frac{1}{\sqrt{2}} \Lambda_{d} v_{d} \\
\frac{1}{\sqrt{2}} g_{2} v_{d} & +\mu_{d}^{\text {eff, }}
\end{array}\right)
$$


The diagonalization and transformation to mass eigenstates $\lambda_{i}^{ \pm}$is performed by two unitary matrices $U^{1}$ and $V^{1}$ as

$$
\begin{array}{rlrl}
U^{1, *} m_{\chi^{+}} V^{1, \dagger}=m_{\chi^{+}}^{\operatorname{diag}}, & \tilde{T}^{-}=\sum_{j=1}^{2} U_{j 1}^{1, *} \lambda_{j}^{-}, & \tilde{H}_{d}^{-}=\sum_{j=1}^{2} U_{j 2}^{1, *} \lambda_{j}^{-}, \\
\tilde{W}^{+}=\sum_{j=1}^{2} V_{1 j}^{1, *} \lambda_{j}^{+}, & R_{d}^{+}=\sum_{j=1}^{2} V_{2 j}^{1, *} \lambda_{j}^{+}
\end{array}
$$

and the corresponding physical four-component charginos are built as

$$
\chi_{i}^{+}=\left(\begin{array}{c}
\lambda_{i}^{+} \\
\lambda_{i}^{-*}
\end{array}\right) \quad i=1,2 .
$$

The second submatrix, in the basis $\left(\tilde{W}^{-}, R_{u}^{-}\right),\left(\tilde{T}^{+}, \tilde{H}_{u}^{+}\right)$of spinors with R-charge equal to minus electric charge, reads

$$
m_{\rho^{-}}=\left(\begin{array}{cc}
-g_{2} v_{T}+M_{W}^{D} & \frac{1}{\sqrt{2}} g_{2} v_{u} \\
-\frac{1}{\sqrt{2}} \Lambda_{u} v_{u} & -\mu_{u}^{\text {eff, }+}
\end{array}\right)
$$

The diagonalization and transformation to mass eigenstates $\eta_{i}^{ \pm}$is performed by $U^{2}$ and $V^{2}$ as

$$
\begin{aligned}
& U^{2, *} m_{\rho^{-}} V^{2, \dagger}=m_{\rho^{-}}^{\operatorname{diag}}, \quad \tilde{W}^{-}=\sum_{j=1}^{2} U_{j 1}^{2, *} \eta_{j}^{-}, \quad R_{u}^{-}=\sum_{j=1}^{2} U_{j 2}^{2, *} \eta_{j}^{-} \\
& \tilde{T}^{+}=\sum_{j=1}^{2} V_{1 j}^{2, *} \eta_{j}^{+}, \quad \tilde{H}_{u}^{+}=\sum_{j=1}^{2} V_{2 j}^{2, *} \eta_{j}^{+}
\end{aligned}
$$

and the other two physical four-component charginos are built as

$$
\rho_{i}^{-}=\left(\begin{array}{c}
\eta_{i}^{-} \\
\eta_{i}^{+*}
\end{array}\right) \quad i=1,2 .
$$

Open Access. This article is distributed under the terms of the Creative Commons Attribution License (CC-BY 4.0), which permits any use, distribution and reproduction in any medium, provided the original author(s) and source are credited.

\section{References}

[1] ATLAS collaboration, Observation of a new particle in the search for the Standard Model Higgs boson with the ATLAS detector at the LHC, Phys. Lett. B 716 (2012) 1 [arXiv: 1207.7214] [INSPIRE].

[2] CMS collaboration, Observation of a new boson at a mass of $125 \mathrm{GeV}$ with the CMS experiment at the LHC, Phys. Lett. B 716 (2012) 30 [arXiv:1207.7235] [INSPIRE]. 
[3] ATLAS and CMS collaborations, Measurements of the Higgs boson production and decay rates and constraints on its couplings from a combined ATLAS and CMS analysis of the LHC pp collision data at $\sqrt{s}=7$ and 8 TeV, ATLAS-CONF-2015-044 (2015).

[4] S. Heinemeyer, O. Stal and G. Weiglein, Interpreting the LHC Higgs Search Results in the MSSM, Phys. Lett. B 710 (2012) 201 [arXiv:1112.3026] [INSPIRE].

[5] R. Benbrik, M. Gomez Bock, S. Heinemeyer, O. Stal, G. Weiglein and L. Zeune, Confronting the MSSM and the NMSSM with the Discovery of a Signal in the two Photon Channel at the LHC, Eur. Phys. J. C 72 (2012) 2171 [arXiv:1207.1096] [InSPIRE].

[6] P. Bechtle, S. Heinemeyer, O. Stal, T. Stefaniak, G. Weiglein and L. Zeune, MSSM Interpretations of the LHC Discovery: Light or Heavy Higgs?, Eur. Phys. J. C 73 (2013) 2354 [arXiv: 1211.1955] [INSPIRE].

[7] U. Ellwanger, C. Hugonie and A.M. Teixeira, The Next-to-Minimal Supersymmetric Standard Model, Phys. Rept. 496 (2010) 1 [arXiv:0910.1785] [InSPIRE].

[8] M. Maniatis, The Next-to-Minimal Supersymmetric extension of the Standard Model reviewed, Int. J. Mod. Phys. A 25 (2010) 3505 [arXiv:0906.0777] [InSPIRE].

[9] Z. Kang, J. Li and T. Li, On Naturalness of the MSSM and NMSSM, JHEP 11 (2012) 024 [arXiv: 1201.5305] [INSPIRE].

[10] D.A. Vasquez, G. Bélanger, C. Boehm, J. Da Silva, P. Richardson and C. Wymant, The $125 \mathrm{GeV}$ Higgs in the NMSSM in light of LHC results and astrophysics constraints, Phys. Rev. D 86 (2012) 035023 [arXiv: 1203.3446] [INSPIRE].

[11] G. Bélanger, U. Ellwanger, J.F. Gunion, Y. Jiang, S. Kraml and J.H. Schwarz, Higgs Bosons at 98 and $125 \mathrm{GeV}$ at LEP and the LHC, JHEP 01 (2013) 069 [arXiv:1210.1976] [INSPIRE].

[12] S.F. King, M. Mühlleitner, R. Nevzorov and K. Walz, Natural NMSSM Higgs Bosons, Nucl. Phys. B 870 (2013) 323 [arXiv:1211.5074] [INSPIRE].

[13] M. Badziak, M. Olechowski and S. Pokorski, New Regions in the NMSSM with a $125 \mathrm{GeV}$ Higgs, JHEP 06 (2013) 043 [arXiv: 1304.5437] [INSPIRE].

[14] N.D. Christensen, T. Han, Z. Liu and S. Su, Low-Mass Higgs Bosons in the NMSSM and Their LHC Implications, JHEP 08 (2013) 019 [arXiv:1303.2113] [INSPIRE].

[15] R. Barbieri, D. Buttazzo, K. Kannike, F. Sala and A. Tesi, One or more Higgs bosons?, Phys. Rev. D 88 (2013) 055011 [arXiv: 1307.4937] [inSPIRE].

[16] J. Cao, F. Ding, C. Han, J.M. Yang and J. Zhu, A light Higgs scalar in the NMSSM confronted with the latest LHC Higgs data, JHEP 11 (2013) 018 [arXiv:1309.4939] [INSPIRE].

[17] U. Ellwanger and C. Hugonie, The semi-constrained NMSSM satisfying bounds from the LHC, LUX and Planck, JHEP 08 (2014) 046 [arXiv: 1405.6647] [INSPIRE].

[18] K. Huitu and H. Waltari, Higgs sector in NMSSM with right-handed neutrinos and spontaneous R-parity violation, JHEP 11 (2014) 053 [arXiv: 1405.5330] [INSPIRE].

[19] S.F. King, M. Mühlleitner, R. Nevzorov and K. Walz, Discovery Prospects for NMSSM Higgs Bosons at the High-Energy Large Hadron Collider, Phys. Rev. D 90 (2014) 095014 [arXiv: 1408.1120] [INSPIRE].

[20] K.S. Jeong, Y. Shoji and M. Yamaguchi, Higgs Mixing in the NMSSM and Light Higgsinos, JHEP 11 (2014) 148 [arXiv:1407.0955] [INSPIRE].

[21] M. Guchait and J. Kumar, Light Higgs Bosons in NMSSM at the LHC, arXiv: 1509.02452 [INSPIRE]. 
[22] A. Bottino, N. Fornengo and S. Scopel, Phenomenology of light neutralinos in view of recent results at the CERN Large Hadron Collider, Phys. Rev. D 85 (2012) 095013 [arXiv:1112.5666] [INSPIRE].

[23] C. Boehm, P.S.B. Dev, A. Mazumdar and E. Pukartas, Naturalness of Light Neutralino Dark Matter in pMSSM after LHC, XENON100 and Planck Data, JHEP 06 (2013) 113 [arXiv: 1303.5386] [INSPIRE].

[24] G. Bélanger, G. Drieu La Rochelle, B. Dumont, R.M. Godbole, S. Kraml and S. Kulkarni, LHC constraints on light neutralino dark matter in the MSSM, Phys. Lett. B 726 (2013) 773 [arXiv: 1308.3735] [INSPIRE].

[25] A. Arbey, M. Battaglia and F. Mahmoudi, Supersymmetry with Light Dark Matter confronting the recent CDMS and LHC Results, Phys. Rev. D 88 (2013) 095001 [arXiv: 1308.2153] [INSPIRE].

[26] J. Cao, C. Han, L. Wu, P. Wu and J.M. Yang, A light SUSY dark matter after CDMS-II, LUX and LHC Higgs data, JHEP 05 (2014) 056 [arXiv:1311.0678] [INSPIRE].

[27] C. Beskidt, W. de Boer and D.I. Kazakov, The impact of a $126 \mathrm{GeV}$ Higgs on the neutralino mass, Phys. Lett. B 738 (2014) 505 [arXiv: 1402.4650] [INSPIRE].

[28] T. Han, Z. Liu and S. Su, Light Neutralino Dark Matter: Direct/Indirect Detection and Collider Searches, JHEP 08 (2014) 093 [arXiv: 1406.1181] [INSPIRE].

[29] G.D. Kribs, E. Poppitz and N. Weiner, Flavor in supersymmetry with an extended R-symmetry, Phys. Rev. D 78 (2008) 055010 [arXiv:0712.2039] [INSPIRE].

[30] A. Salam and J.A. Strathdee, Supersymmetry and Fermion Number Conservation, Nucl. Phys. B 87 (1975) 85 [INSPIRE].

[31] P. Fayet, Supergauge Invariant Extension of the Higgs Mechanism and a Model for the electron and Its Neutrino, Nucl. Phys. B 90 (1975) 104 [INSPIRE].

[32] E. Dudas, M. Goodsell, L. Heurtier and P. Tziveloglou, Flavour models with Dirac and fake gluinos, Nucl. Phys. B 884 (2014) 632 [arXiv:1312.2011] [INSPIRE].

[33] G.D. Kribs and A. Martin, Supersoft Supersymmetry is Super-Safe, Phys. Rev. D 85 (2012) 115014 [arXiv: 1203.4821] [INSPIRE].

[34] G. Bélanger, K. Benakli, M. Goodsell, C. Moura and A. Pukhov, Dark Matter with Dirac and Majorana Gaugino Masses, JCAP 08 (2009) 027 [arXiv:0905.1043] [INSPIRE].

[35] E.J. Chun, J.-C. Park and S. Scopel, Dirac gaugino as leptophilic dark matter, JCAP 02 (2010) 015 [arXiv:0911.5273] [INSPIRE].

[36] M.R. Buckley, D. Hooper and J. Kumar, Phenomenology of Dirac Neutralino Dark Matter, Phys. Rev. D 88 (2013) 063532 [arXiv:1307.3561] [InSPIRE].

[37] P.J. Fox, A.E. Nelson and N. Weiner, Dirac gaugino masses and supersoft supersymmetry breaking, JHEP 08 (2002) 035 [hep-ph/0206096] [INSPIRE].

[38] T. Plehn and T.M.P. Tait, Seeking Sgluons, J. Phys. G 36 (2009) 075001 [arXiv:0810.3919] [INSPIRE].

[39] S.Y. Choi, M. Drees, J. Kalinowski, J.M. Kim, E. Popenda and P.M. Zerwas, Color-Octet Scalars of $N=2$ Supersymmetry at the LHC, Phys. Lett. B 672 (2009) 246 [arXiv: 0812.3586] [INSPIRE].

[40] S.Y. Choi, D. Choudhury, A. Freitas, J. Kalinowski, J.M. Kim and P.M. Zerwas, Dirac Neutralinos and Electroweak Scalar Bosons of $N=1 / N=2$ Hybrid Supersymmetry at Colliders, JHEP 08 (2010) 025 [arXiv: 1005.0818] [INSPIRE]. 
[41] S.Y. Choi, D. Choudhury, A. Freitas, J. Kalinowski and P.M. Zerwas, The Extended Higgs System in R-symmetric Supersymmetry Theories, Phys. Lett. B 697 (2011) 215 [Erratum ibid. B 698 (2011) 457] [arXiv: 1012.2688] [INSPIRE].

[42] K. Benakli, M.D. Goodsell and F. Staub, Dirac Gauginos and the 125 GeV Higgs, JHEP 06 (2013) 073 [arXiv:1211.0552] [INSPIRE].

[43] ATLAS collaboration, Search for pair-produced massive coloured scalars in four-jet final states with the ATLAS detector in proton-proton collisions at $\sqrt{s}=7 \mathrm{TeV}$, Eur. Phys. J. C 73 (2013) 2263 [arXiv: 1210.4826] [INSPIRE].

[44] ATLAS collaboration, Search for anomalous production of events with same-sign dileptons and $b$ jets in $14.3 \mathrm{fb}^{-1}$ of $\mathrm{pp}$ collisions at $\sqrt{\mathrm{s}}=8 \mathrm{TeV}$ with the ATLAS detector, ATLAS-CONF-2013-051 (2013).

[45] W. Kotlarski, A. Kalinowski and J. Kalinowski, Searching for Sgluons in the Same-sign Leptons Final State at the LHC, Acta Phys. Polon. B 44 (2013) 2149.

[46] P. Dießner, J. Kalinowski, W. Kotlarski and D. Stöckinger, Higgs boson mass and electroweak observables in the MRSSM, JHEP 12 (2014) 124 [arXiv:1410.4791] [INSPIRE].

[47] P. Diessner, J. Kalinowski, W. Kotlarski and D. Stöckinger, Two-loop correction to the Higgs boson mass in the MRSSM, Adv. High Energy Phys. 2015 (2015) 760729 [arXiv: 1504.05386] [INSPIRE].

[48] E. Bertuzzo, C. Frugiuele, T. Gregoire and E. Ponton, Dirac gauginos, $R$ symmetry and the $125 \mathrm{GeV}$ Higgs, JHEP 04 (2015) 089 [arXiv:1402.5432] [INSPIRE].

[49] S.P. Martin, Nonstandard supersymmetry breaking and Dirac gaugino masses without supersoftness, Phys. Rev. D 92 (2015) 035004 [arXiv:1506.02105] [INSPIRE].

[50] Wolfram Research, Inc., Mathematica Version 9.0/10.0, Champaign, IL U.S.A. (2012-2014).

[51] F. Staub, SARAH, arXiv:0806.0538 [INSPIRE].

[52] F. Staub, From Superpotential to Model Files for FeynArts and CalcHep/CompHEP, Comput. Phys. Commun. 181 (2010) 1077 [arXiv:0909. 2863] [INSPIRE].

[53] F. Staub, Automatic Calculation of supersymmetric Renormalization Group Equations and Self Energies, Comput. Phys. Commun. 182 (2011) 808 [arXiv: 1002.0840] [INSPIRE].

[54] F. Staub, SARAH 3.2: Dirac Gauginos, UFO output and more, Comput. Phys. Commun. 184 (2013) 1792 [arXiv: 1207.0906] [InSPIRE].

[55] F. Staub, SARAH 4: A tool for (not only SUSY) model builders, Comput. Phys. Commun. 185 (2014) 1773 [arXiv: 1309.7223] [INSPIRE].

[56] M.D. Goodsell, K. Nickel and F. Staub, Two-Loop Higgs mass calculations in supersymmetric models beyond the MSSM with SARAH and SPheno, Eur. Phys. J. C 75 (2015) 32 [arXiv:1411.0675] [INSPIRE].

[57] W. Porod, SPheno, a program for calculating supersymmetric spectra, SUSY particle decays and SUSY particle production at $e^{+} e^{-}$colliders, Comput. Phys. Commun. 153 (2003) 275 [hep-ph/0301101] [INSPIRE].

[58] W. Porod and F. Staub, SPheno 3.1: Extensions including flavour, CP-phases and models beyond the MSSM, Comput. Phys. Commun. 183 (2012) 2458 [arXiv:1104.1573] [INSPIRE].

[59] P. Athron, J.-h. Park, D. Stöckinger and A. Voigt, FlexibleSUSY - A spectrum generator generator for supersymmetric models, Comput. Phys. Commun. 190 (2015) 139 [arXiv:1406.2319] [INSPIRE]. 
[60] B.C. Allanach, SOFTSUSY: a program for calculating supersymmetric spectra, Comput. Phys. Commun. 143 (2002) 305 [hep-ph/0104145] [INSPIRE].

[61] B.C. Allanach, P. Athron, L.C. Tunstall, A. Voigt and A.G. Williams, Next-to-Minimal SOFTSUSY, Comput. Phys. Commun. 185 (2014) 2322 [arXiv:1311.7659] [InSPIRE].

[62] T. Hahn, Generating Feynman diagrams and amplitudes with FeynArts 3, Comput. Phys. Commun. 140 (2001) 418 [hep-ph/0012260] [INSPIRE].

[63] B. Chokoufe Nejad, T. Hahn, J.N. Lang and E. Mirabella, FormCalc 8: Better Algebra and Vectorization, J. Phys. Conf. Ser. 523 (2014) 012050 [arXiv: 1310.0274] [InSPIRE].

[64] T. Hahn and M. Pérez-Victoria, Automatized one loop calculations in four-dimensions and D-dimensions, Comput. Phys. Commun. 118 (1999) 153 [hep-ph/9807565] [InSPIRE].

[65] P. Bechtle, O. Brein, S. Heinemeyer, G. Weiglein and K.E. Williams, HiggsBounds: Confronting Arbitrary Higgs Sectors with Exclusion Bounds from LEP and the Tevatron, Comput. Phys. Commun. 181 (2010) 138 [arXiv:0811.4169] [INSPIRE].

[66] P. Bechtle, O. Brein, S. Heinemeyer, G. Weiglein and K.E. Williams, HiggsBounds 2.0.0: Confronting Neutral and Charged Higgs Sector Predictions with Exclusion Bounds from LEP and the Tevatron, Comput. Phys. Commun. 182 (2011) 2605 [arXiv:1102.1898] [InSPIRE].

[67] P. Bechtle et al., Recent Developments in HiggsBounds and a Preview of HiggsSignals, PoS (CHARGED 2012) 024 [arXiv: 1301.2345] [INSPIRE].

[68] P. Bechtle et al., HiggsBounds - 4: Improved Tests of Extended Higgs Sectors against Exclusion Bounds from LEP, the Tevatron and the LHC, Eur. Phys. J. C 74 (2014) 2693 [arXiv: 1311.0055] [INSPIRE].

[69] P. Bechtle, S. Heinemeyer, O. Stål, T. Stefaniak and G. Weiglein, HiggsSignals: Confronting arbitrary Higgs sectors with measurements at the Tevatron and the LHC, Eur. Phys. J. C 74 (2014) 2711 [arXiv: 1305.1933] [INSPIRE].

[70] O. Stål and T. Stefaniak, Constraining extended Higgs sectors with HiggsSignals, PoS (EPS-HEP 2013) 314 [arXiv: 1310.4039] [INSPIRE].

[71] T. Robens and T. Stefaniak, Status of the Higgs Singlet Extension of the Standard Model after LHC Run 1, Eur. Phys. J. C 75 (2015) 104 [arXiv: 1501.02234] [INSPIRE].

[72] F. Domingo and G. Weiglein, NMSSM interpretations of the observed Higgs signal, arXiv: 1509.07283 [INSPIRE].

[73] ATLAS collaboration, Search for direct production of charginos, neutralinos and sleptons in final states with two leptons and missing transverse momentum in pp collisions at $\sqrt{s}=8 \mathrm{TeV}$ with the ATLAS detector, JHEP 05 (2014) 071 [arXiv: 1403.5294] [InSPIRE].

[74] CMS collaboration, Searches for electroweak neutralino and chargino production in channels with Higgs, $Z$ and $W$ bosons in pp collisions at 8 TeV, Phys. Rev. D 90 (2014) 092007 [arXiv: 1409.3168] [INSPIRE].

[75] ATLAS collaboration, Search for the direct production of charginos, neutralinos and staus in final states with at least two hadronically decaying taus and missing transverse momentum in pp collisions at $\sqrt{s}=8 \mathrm{TeV}$ with the ATLAS detector, JHEP 10 (2014) 096 [arXiv: 1407.0350] [INSPIRE].

[76] C. Degrande, C. Duhr, B. Fuks, D. Grellscheid, O. Mattelaer and T. Reiter, UFO - The Universal FeynRules Output, Comput. Phys. Commun. 183 (2012) 1201 [arXiv:1108.2040] [INSPIRE]. 
[77] M. Bahr et al., HERWIG++ Physics and Manual, Eur. Phys. J. C 58 (2008) 639 [arXiv: 0803.0883] [INSPIRE].

[78] J. Bellm et al., HERWIG++ 2.7 Release Note, arXiv:1310.6877 [InSPIRE].

[79] M.H. Seymour and A. Siodmok, Constraining MPI models using $\sigma_{\mathrm{eff}}$ and recent Tevatron and LHC Underlying Event data, JHEP 10 (2013) 113 [arXiv:1307.5015] [INSPIRE].

[80] A. Sherstnev and R.S. Thorne, Parton Distributions for LO Generators, Eur. Phys. J. C 55 (2008) 553 [arXiv:0711.2473] [INSPIRE].

[81] M. Drees, H. Dreiner, D. Schmeier, J. Tattersall and J.S. Kim, CheckMATE: Confronting your Favourite New Physics Model with LHC Data, Comput. Phys. Commun. 187 (2014) 227 [arXiv: 1312.2591] [INSPIRE].

[82] DELPHES 3 collaboration, J. de Favereau et al., DELPHES 3, A modular framework for fast simulation of a generic collider experiment, JHEP 02 (2014) 057 [arXiv:1307.6346] [INSPIRE].

[83] M. Cacciari, G.P. Salam and G. Soyez, FastJet User Manual, Eur. Phys. J. C 72 (2012) 1896 [arXiv: 1111.6097] [INSPIRE].

[84] M. Cacciari and G.P. Salam, Dispelling the $N^{3}$ myth for the $k_{t}$ jet-finder, Phys. Lett. B 641 (2006) 57 [hep-ph/0512210] [INSPIRE].

[85] M. Cacciari, G.P. Salam and G. Soyez, The Anti-kt jet clustering algorithm, JHEP 04 (2008) 063 [arXiv: 0802.1189] [INSPIRE].

[86] A.L. Read, Presentation of search results: The CL technique, J. Phys. G 28 (2002) 2693 [INSPIRE].

[87] ATLAS collaboration, Search for direct-slepton and direct-chargino production in final states with two opposite-sign leptons, missing transverse momentum and no jets in 20/fb of pp collisions at $\sqrt{s}=8 \mathrm{TeV}$ with the ATLAS detector, ATLAS-CONF-2013-049 (2013).

[88] ATLAS collaboration, Search for direct production of charginos and neutralinos in events with three leptons and missing transverse momentum in $21 \mathrm{fb}^{-1}$ of $\mathrm{pp}$ collisions at $\sqrt{s}=8 \mathrm{TeV}$ with the ATLAS detector, ATLAS-CONF-2013-035 (2013).

[89] ATLAS collaboration, Search for direct production of charginos and neutralinos in events with three leptons and missing transverse momentum in $\sqrt{s}=8 \mathrm{TeV}$ pp collisions with the ATLAS detector, JHEP 04 (2014) 169 [arXiv:1402.7029] [INSPIRE].

[90] ATLAS collaboration, Search for supersymmetry in events with four or more leptons in $21 \mathrm{fb}^{-1}$ of pp collisions at $\sqrt{s}=8 \mathrm{TeV}$ with the ATLAS detector, ATLAS-CONF-2013-036 (2013).

[91] J. Alwall, R. Frederix, S. Frixione, V. Hirschi, F. Maltoni, O. Mattelaer et al., The automated computation of tree-level and next-to-leading order differential cross sections and their matching to parton shower simulations, JHEP 07 (2014) 079 [arXiv:1405.0301] [INSPIRE].

[92] A. Belyaev, N.D. Christensen and A. Pukhov, CalcHEP 3.4 for collider physics within and beyond the Standard Model, Comput. Phys. Commun. 184 (2013) 1729 [arXiv:1207.6082] [INSPIRE].

[93] ATLAS collaboration, Search for direct pair production of a chargino and a neutralino decaying to the $125 \mathrm{GeV}$ Higgs boson in $\sqrt{s}=8 \mathrm{TeV}$ pp collisions with the ATLAS detector, Eur. Phys. J. C 75 (2015) 208 [arXiv:1501.07110] [InSPIRE].

[94] G. Bélanger, F. Boudjema, A. Pukhov and A. Semenov, MicrOMEGAs4.1: two dark matter candidates, Comput. Phys. Commun. 192 (2015) 322 [arXiv:1407.6129] [INSPIRE]. 
[95] C. Savage, A. Scaffidi, M. White and A.G. Williams, LUX likelihood and limits on spin-independent and spin-dependent WIMP couplings with LUXCalc, Phys. Rev. D 92 (2015) 103519 [arXiv: 1502.02667] [INSPIRE].

[96] LUX collaboration, D.S. Akerib et al., The Large Underground Xenon (LUX) Experiment, Nucl. Instrum. Meth. A 704 (2013) 111 [arXiv:1211.3788] [INSPIRE].

[97] Particle Data Group collaboration, K.A. Olive et al., Review of Particle Physics, Chin. Phys. C 38 (2014) 090001 [inSPIRE].

[98] Planck collaboration, P.A.R. Ade et al., Planck 2013 results. XVI. Cosmological parameters, Astron. Astrophys. 571 (2014) A16 [arXiv:1303.5076] [INSPIRE]. 